\title{
Continuous Stirred Tank Reactors: easy to stabilise?
}

\author{
R. Antonelli \\ Centre for Process Systems Engineering \\ Imperial College of Science, Technology and Medicine \\ London, SW7 2BY, UK \\ A. Astolfi \\ Dept. of Electrical and Electronic Engineering \\ Imperial College of Science, Technology and Medicine \\ London, SW7 2BT, UK \\ and \\ Dipartimento di Elettronica e Informazione \\ Politecnico di Milano \\ Milano, ITALY
}

April 4, 2001

\footnotetext{
*Author to whom correspondence should be addressed. Fax:+44-20-7594-6282, Email:a.astolfi@ic.ac.uk.
} 


\begin{abstract}
The objective of this work is to make use of methodologies and tools from nonlinear Lyapunov theory in the design of bounded control laws for the temperature stabilisation of Continuous Stirred Tank Reactors (CSTRs) with exothermic or endothermic reactions. Simple output feedback control laws, i.e. control laws that feed back only the available measurements, are designed. It is shown that these controllers yield Global Asymptotic Stability (GAS) and do not require precise knowledge of the system parameters. The capabilities of the proposed method to handle control input constraints and model uncertainty are also discussed. The work is then completed with the analysis of two case studies: the classical first order irreversible reaction $A \rightarrow B$ and the more complex van der Vusse reaction mechanism.
\end{abstract}

Keywords: Chemical Reactors, Temperature Stabilisation, Input Constraints, Lyapunov-Based Control, Bounded Output Feedback Control. 


\section{Introduction}

Many processes of industrial interest are difficult to control for their inherent nonlinear behaviour, presence of input constraints and lack of measurements. Among these systems, chemical processes have been widely studied and traditionally controlled using linear system analysis and design tools together with linearised models [11,32,34], or nonlinear methods based on linearisation techniques $[3,5,16,17,22,29,31]$. However, the use of linear techniques is very limiting if the analysed chemical process is highly nonlinear, whereas nonlinear control design needs, in general, too much information about the process.

Chemical reactors exhibit a challenging control problem due to their nonlinearity, chaotic behaviour and the presence of several stable and unstable equilibrium points. The uncertainty affecting the kinetic parameters is also not negligible: modelling of kinetic reactions is difficult and, as a result, the model mismatch may be significant. If the controller design utilises too much information, the control performance may be severely degraded. Furthermore, most nonlinear control techniques assume that all state variables are measured or accurately estimated. In industrial practice, the estimation of unmeasured state variables and unknown parameters is not a trivial problem. Usually, only the temperatures and flowrates are easily measurable and, although advanced methods for accurate measurement of the concentrations in a chemical reactor have been developed, these have not been used frequently in industrial plants because of very high operating costs. Finally, the hard constraints imposed on the control input may adversely affect the efficiency of the controller and degrade the overall performance of the control system.

During the past decade there has been a significant increase in interest by many industries in the study of appropriate control strategies for batch, semi-batch or continuous reactors. Several researchers have studied the reactor control problem $[1,2,3,4,5,6,7,11,13,15,16,17,19,23,24,28,29,30,31$, $35,36]$.

Conventional Proportional-Integral-Derivative (PID) controllers have been first applied for the temperature regulation of chemical reactors. They are usu-

ally robust to model uncertainty since their design is partly independent from the model of the system, but dependent on tuning. The temperature stabilisation of an open stirred tank reactor with a first order exothermic reaction was extensively studied using a proportional controller [11]. This process shows 
three open loop steady states, two of which are stable and one unstable. Aris and Amundson [11], studied the dynamic behaviour of a reactor and used the linearisation method to show that the open loop unstable equilibrium point becomes locally asymptotically stable in closed loop.

Feedback linearisation techniques have also been used. In particular, a full state feedback linearisation solution is presented for an exothermic reactor with one reaction [3, 31]. Global stabilisation by state feedback has been numerically demonstrated using the coolant temperature as a manipulated variable and the addition of a state observer [31]. Stabilisation by state feedback has been proved with respect to a well defined domain [3]. Input-output linearising control, an analytical design approach which aims to reduce the original nonlinear control problem to a simpler linear control problem, has been extensively investigated $[15,30]$. An input-output linearisation strategy for constrained nonlinear processes is proposed by combining the benefits of feedback linearisation and model predictive control [30], but robustness to model uncertainty is not guaranteed since feedback linearisation techniques require exact knowledge of the process model.

Various solutions have been proposed to overcome this problem, in particular the addition of an integral action to the controller obtained from input-output linearisation has been proposed [29]. Lyapunov-based adaptive linearising techniques have been successively developed $[1,2,28]$. First, global stabilisation by state feedback of a reactor with an exothermic reaction was proved [1]. Then, the same case was considered in presence of a state observer [2]. Finally, global stabilisation of a reactor with complex kinetics was dealt with [28], still considering a state observer. Good control performance was obtained with a state observer in the case of a reactor with two sequential exothermic reactions [16].

Important studies have been made by Alvarez et al. [3], who used nonlinear geometric control tools to stabilise unstable steady states of a CSTR and considered the performance of Proportional (P) and Proportional-Integral (PI) controllers. Lately, they studied the behavior of nonlinear controllers for the specific case of an exothermic, first order, irreversible reactor [4]. Moreover, they showed the global boundedness of trajectories and the practical stabilisation of a chemical reactor when driven by an input-output linearising type feedback, under parametric uncertainty [5]. Finally, they proved that a CSTR with unknown reaction rates can be stabilised by applying a high-gain feedback law and introduced a state observer to solve the problem of input saturation 
and poor closed loop performance [7].

Robust control techniques have been applied to a reactor with a first order exothermic reaction $[5,13,17]$. Semi-global practical stabilisation has been proved in the case of parametric uncertainty on the kinetics by means of a robust input-state linearising state feedback [13]. In recent years, interesting attempts to include the process nonlinearities into an adaptive controller have been made with application to a two sequential reactions: the zero dynamics are analysed and a model reference adaptive controller is designed to control a highly nonlinear CSTR [2, 16].

Still recently, some significant results were also obtained for the robust global stabilisation under input constraints. Viel et al. [36] designed a state feedback controller to stabilise the temperature at an arbitrary set-point in spite of uncertainties on the reaction kinetics and proposed a robust state observer to overcome the problem of partial state measurement. Then they extended these results to an exothermic chemical reactor considering the feed concentration as additional input [35]. Another approach has been proposed by Ortega et al. [19]. Therein a semi-adaptive state feedback controller was applied in the region of the state space where convexity can be used to reduce parameter uncertainty and it is then frozen and switched to a robust scheme outside this region.

It must be noted that to be implemented, all the above control laws need the on-line measurement of the full state of the system, represented by the set of concentrations and the temperatures. In industrial practice it is very easy to measure temperatures, but usually not concentrations.

The problem of state variable measurement or estimation can be overcome if we select an output feedback controller $[8,9,10]$ able to regulate and globally stabilise the reactor temperature at its set-point without requiring any knowledge of the concentrations. Interesting results in this direction have been obtained $[6,23,24]$. A first order compensator with modelling error compensation is designed and its ability to stabilise the temperature of a chemical reactor is proved [6]. Then, the robust stabilisation of a class of CSTRs with unknown kinetics and control input saturations is achieved by means of modelling error compensation techniques which consist of interpreting the modelling error signal as a new state, whose dynamics are observable from output measurements [24]. Finally the stabilisation of chemical reactors by output feedback with PI-type controllers has been reviewed and treated in detail [23].

The objective of this work is to use Lyapunov-based methods to design non- 
linear output feedback control laws for the temperature stabilisation of a wide class of chemical reactors. The proposed controllers yield robust stability and guaranteed performance also in the presence of input constraints and uncertainty on the kinetic parameters. The paper is organised as follows. Section 2 briefly discusses the main features of chemical reactors and, in particular, focuses on the dynamic model of non-isothermal continuous stirred tank reactors. Section 3 and Section 4 are respectively concerned with the problem statement, some preliminary assumptions and an introductory lemma used to derive the main results presented in Section 5, i.e. dynamic output feedback stabilising controllers for single and multi-input systems. Section 6 presents some simulations results based on two case studies: a simple irreversible first order reaction and the reaction mechanism known as van der Vusse reaction. Finally Section 7 discusses the main goal achieved in the paper giving some conclusions.

\section{Dynamics of Chemical Reactors}

Consider a reactor where a set of $m>0$ independent chemical reactions take place between $n>0$ chemical species. An external jacket is used to remove or supply heat to the system. In general, a reaction mechanism taking place in a stirred tank can be expressed in the form [21]

$$
\sum_{j=1}^{n} \nu_{j i} A_{j}=0 \quad i=1, \ldots, m
$$

where $A_{j}$ denotes the $j$-th chemical species and $\nu_{j i}$ is its stoichiometric coefficient in the $i$-th reaction. The component $A_{j}$ is defined reactant of the reaction $i$ if $\nu_{j i}<0$ and product of the reaction $i$ if $\nu_{j i}>0$. The information contained in the reaction scheme (1) can be summarized into a matrix $C$, called the characteristic matrix, in which the elements are the stoichiometric coefficients of reactants and products. As a matter of illustration, the reaction

$$
2 A_{1}+A_{2} \longrightarrow A_{3}
$$

can be rewritten as

$$
-2 A_{1}-A_{2}+A_{3}=0
$$

yielding the characteristic matrix

$$
C=\left(\begin{array}{r}
-2 \\
-1 \\
1
\end{array}\right)
$$


From a dynamic viewpoint, in chemical reactions, the actual reacted concentration of the generic component $A_{j}$ can be determined by evaluating the reaction rate, which may have a different numerical value according to the way it is defined and measured. In general, the reaction rate is a nonlinear function of the temperature $T$, usually expressed in Kelvin, and of the vector $x^{r}$ of the concentrations of the reacting species. It expresses the rate of reactant consumption and product formation in the reactor. Usually the reaction rate is non-negative, possibly a time-varying function. Just for simplicity, this work will consider the case where the reaction rate vector $r\left(x^{r}, T\right)$ depends only upon $x^{r}$ and $T$ and is separable with respect to its arguments, i.e.

$$
r\left(x^{r}, T\right)=\varphi\left(x^{r}\right) k(T)
$$

where the vector $k(T) \in \mathbb{R}^{m}$ is called rate constant of reaction and it is composed of positive bounded functions of the temperature, while $\varphi\left(x^{r}\right) \in \mathbb{R} \mathbb{R}^{m \times m}$ is a diagonal matrix whose elements are functions of the vector $x^{r}$ of the concentrations of the reactants. This is related to the vector $x$ of the $n$ concentrations of chemical species involved in the reaction network (reactants and products), by a projection operator, i.e.

$$
x_{i}^{r}=P_{i}^{r} x
$$

where $P_{i}^{r}$ is the operator that projects the concentrations of the reactants of the $i$-th reaction into the vector $x_{i}^{r}$, such that $x^{r}=\left[x_{1}^{r}, \ldots, x_{m}^{r}\right] \in \mathbb{R} R^{m}$. In matrix form, one has $P_{i}^{r}=\operatorname{diag}(0, \ldots, 1,0,1, \ldots, 1, \ldots, 0)$. Moreover, note that the vector $k(T)$ is a continuous and strictly positive function for all $T>0$ and that

$$
\lim _{T \rightarrow 0^{+}} k(T)=0
$$

in accordance with the thermodynamic principle that a chemical reaction cannot occur at absolute zero. In the common case when the process kinetics follow the Arrhenius law, the reaction rate vector can be expressed as [16]

$$
r\left(x^{r}, T\right)=\left[\begin{array}{cccc}
\varphi_{1}\left(x_{1}^{r}\right) & 0 & \ldots & 0 \\
0 & \varphi_{2}\left(x_{2}^{r}\right) & \ldots & \vdots \\
\vdots & \vdots & \ldots & 0 \\
0 & 0 & \ldots & \varphi_{m}\left(x_{m}^{r}\right)
\end{array}\right]\left[\begin{array}{c}
k_{01} e^{-E_{1} / R T} \\
k_{02} e^{-E_{2} / R T} \\
\vdots \\
k_{0 m} e^{-E_{m} / R T}
\end{array}\right]
$$

where $k_{0 i}$, for $i=1 \ldots m$, are the constant pre-exponential factors, $E_{i}$, for $i=$ $1 \ldots m$, are the activation energies and $R$ is the Joule constant. The functions 
$\varphi_{i}\left(x_{i}^{r}\right)$, for $i=1 \ldots m$, are generally nonlinear functions of the concentration of the reactants involved in the $i$ th reaction, i.e.

$$
\varphi_{i}\left(x_{i}^{r}\right)=\prod_{j \in r} \phi_{i j}\left(x_{j}\right), \quad i=1, \ldots, m
$$

where, in the presence of elementary reactions $[21], \phi_{i j}\left(x_{j}\right)$ represents the mass action law

$$
\phi_{i j}\left(x_{j}\right)=x_{j}^{\nu_{j i}}, \quad \nu_{j i} \in \mathbb{N}
$$

The above considerations are important to better illustrate the main concepts related to the dynamic behaviour of continuous non-isothermal stirred tank reactors, as presented in what follows.

\subsection{Continuous Stirred Tank Reactors}

A type of reactor widely used in industry is a well-stirred tank into which there is a continuous flow of reacting material and from which the reacted or partially reacted material passes continuously. This reactor is referred to as a Continuous Stirred Tank Reactor (CSTR). A CSTR is generally assumed to be homogeneous and therefore modelled as having no spatial variations in concentration, temperature or reaction rate throughout the vessel. In fact, the main assumption concerning the CSTR dynamics is that perfect mixing occurs inside the reactor. This means that the properties of the reaction mixture are considered uniform everywhere within the reaction vessel and therefore identical to the properties of the outlet stream. Another important assumption is that the inlet stream instantaneously mixes with the mixture already inside the reactor. Practically, if an entering element of reacting material is distributed uniformly throughout the tank in a time much shorter than the average residence time in the tank, then the tank can be considered well-mixed. A schematic example of such a chemical reactor, with typical chemical process symbolism [33], is represented in Figure 1.

Continuous processes are adopted in almost all large-scale chemical industries. In general, they imply reduced costs due to the elimination of operations, such as the repeated filling and emptying of batch reactors, and a greater constancy in reaction conditions. However, complex interactions between transport phenomena and reaction kinetics characterise this class of reactors and determine their performance. Modelling of the kinetic mechanism and heat transfer phenomena have been a subject of active research in chemical engineering in 


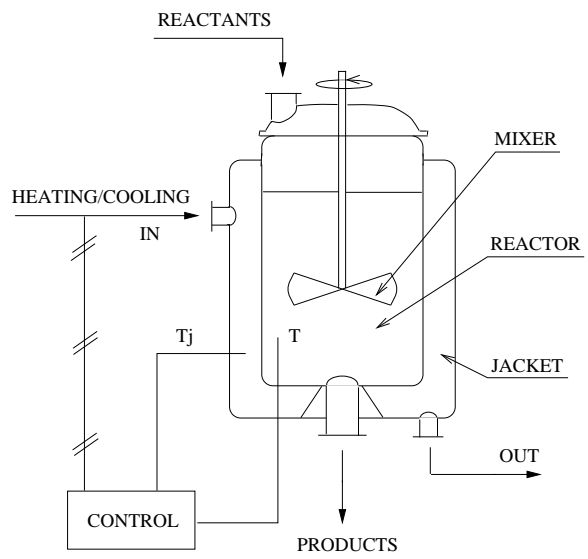

Figure 1: Block scheme of a CSTR. The external jacket is used as a heat source or sink. The reactor temperature $T$ and the jacket temperature $T_{j}$ are controlled by means of the heating or cooling flowrate.

the recent years $[14,18]$. Unfortunately in many cases the structure of the reaction kinetics still continues to be uncertain. The presence of many uncertainties renders ineffective a control scheme that uses too much information about the process. Similarly to other research works in this area (see $e . g .[23,27]$ ), in this paper some assumptions have been introduced in the attempt to reduce the model complexity. In particular we will assume the following.

Hp1) There is a complete uniformity of concentration and temperature within the reactor and a complete uniformity of temperature inside the jacket.

Hp2) The reactor and jacket volumes are constant.

Hp3) The reaction kinetics are $C^{1}$ functions.

$\boldsymbol{H p}$ 4) The thermal exchange between the reactor and the jacket is expressed by a constant global heat exchange coefficient $U$ and the amount of heat retained in the reactor walls is considered negligible.

Hp5) The reaction enthalpies $\Delta H_{R i}$ are independent of temperature, positive for endothermic reactions and negative for exothermic reactions.

Hp6) The heat capacities, $c p_{1}$ and $c p_{2}$, of the process fluids, i.e. reactor content and heat transfer medium, are constant.

Under the above assumptions, the material and energy balances applied to the 
jacket reactor give the following mathematical model [20]:

$$
\left\{\begin{aligned}
\dot{x} & =C r\left(x^{r}, T\right)+\frac{Q}{V_{1}}\left(x^{i n}-x\right) \\
\dot{T} & =H_{r}^{T} r\left(x^{r}, T\right)+\frac{U A_{t}}{\rho_{1} V_{1} c p_{1}}\left(T_{j}-T\right)+\frac{Q}{V_{1}}\left(T^{i n}-T\right) \\
\dot{T}_{j} & =-\frac{U A_{t}}{\rho_{2} V_{2} c p_{2}}\left(T_{j}-T\right)+\frac{D}{V_{2}}\left(T_{j}^{i n}-T_{j}\right)
\end{aligned}\right.
$$

that can be rewritten in compact form as

$$
\left\{\begin{aligned}
\dot{x} & =C r\left(x^{r}, T\right)+d\left(x^{i n}-x\right) \\
\dot{T} & =B\left(x^{r}, T\right)+q_{1}\left(T_{j}-T\right)+d\left(T^{i n}-T\right) \\
\dot{T}_{j} & =-q_{2}\left(T_{j}-T\right)+u
\end{aligned}\right.
$$

where $x \in \mathbb{R}^{n}$ is the vector of the $n$ concentrations of reactants $r$ and products $p$; $x^{r} \in \mathbb{R}^{r}$ is the vector of the $r$ concentrations of reactants; $C \in \mathbb{R}^{n \times m}$ is the characteristic matrix of the stoichiometric coefficients; $r\left(x^{r}, T\right) \in \mathbb{R}^{m}$ is the vector of reaction kinetics, expressed as in equation $(2) ; H_{r}^{T}=\left[\frac{\left(-\Delta H_{R 1}\right)}{\rho_{1} c p_{1}}, \ldots, \frac{\left(-\Delta H_{R m}\right)}{\rho_{m} c p_{m}}\right]=$ $\left[b_{1}, b_{2}, \ldots, b_{m}\right] \in \mathbb{R}^{m}$ is the vector of reaction enthalpies; $T \in \mathbb{R}_{+}$is the reactor temperature; $d \in \mathbb{R}_{+}$is the dilution rate, that is the ratio between the feed stream flowrate and the volume of the reactor; $x^{i n} \in R^{n}$ is the vector of nonnegative reactor feed concentrations; $B\left(x^{r}, T\right)=H_{r}^{T} r\left(x^{r}, T\right) \in \mathbb{R}$ is the global reaction heat; $q_{1} \in \mathbb{R}_{+}$is the side reactor heat transfer parameter; $q_{2} \in \mathbb{R}_{+}$ is the side jacket heat transfer parameter; $T_{j} \in \mathbb{R}_{+}$is the jacket temperature; $T^{i n} \in \mathbb{R}_{+}$is the reactor feed temperature; $T_{j}^{i n} \in \mathbb{R}_{+}$is the jacket fluid stream feed temperature; $u \in \mathbb{R}$ is the manipulated heat. By assumption $\boldsymbol{H p 5}$ ), the components $b_{i}$ of the vector of reaction enthalpies, $H_{r}^{T}$, are negative for endothermic reactions and positive for exothermic reactions. Finally the physical parameters of the model are listed in Table 1.

Let $x_{s s}, T_{s s}, T_{j}^{s s}, u_{s s}$ be steady state values of the state and input variables respectively, and define, as in [6], the deviation variables

$$
\begin{aligned}
\psi & =x-x_{s s} \in \mathbb{R}^{n} \\
\zeta & =T-T_{s s} \in \mathbb{R} \\
\eta & =T_{j}-T_{j}^{s s} \in \mathbb{R} \\
\delta u & =u-u_{s s} \in \mathbb{R} .
\end{aligned}
$$




\begin{tabular}{|l|l|}
\hline Parameter & Description \\
\hline$A_{t}$ & heat exchange area, $\mathrm{m}^{2}$ \\
$V_{1}$ & reactor volume, $\mathrm{m}^{3}$ \\
$V_{2}$ & jacket volume, $\mathrm{m}^{3}$ \\
$c p_{1}$ & reactor content heat capacity, $\mathrm{kJ} / \mathrm{kg}^{\circ} \mathrm{K}$ \\
$c p_{2}$ & heat transfer fluid heat capacity, $\mathrm{kJ} / \mathrm{kg}^{\circ} \mathrm{K}$ \\
$\rho_{1}$ & reactor content density, $\mathrm{kg} / \mathrm{m}^{3}$ \\
$\rho_{2}$ & heat transfer fluid density, $\mathrm{kg} / \mathrm{m}^{3}$ \\
$Q$ & volumetric flowrate of the feed stream, $\mathrm{m}^{3} / \mathrm{min}^{3}$ \\
$D$ & volumetric flowrate of the heat transfer fluid, $\mathrm{m}^{3} / \mathrm{min}$ \\
$U$ & global heat exchange coefficient, $\mathrm{kJ} /{ }^{\circ} \mathrm{Km}^{2} \mathrm{~min}$ \\
$\Delta H_{R i}$ & heat of the $i$-th reaction at temperature $\mathrm{T}, \mathrm{kJ} / \mathrm{mol}$ \\
\hline
\end{tabular}

Table 1: Definition of the physical parameters for the CSTR model (7).

Define

$$
\psi_{i}^{r}=P_{i}^{r} \psi_{i}
$$

where $P_{i}^{r}$ is as in (3), and $\left(\psi^{r}\right)^{T}=\left[\psi_{1}^{r}, \ldots, \psi_{m}^{r}\right] \in \mathbb{R}^{m}$, then the system (8) can be rewritten as

$$
\left\{\begin{array}{l}
\dot{\psi}=C \tilde{r}\left(\psi^{r}, \zeta\right)+d\left(\psi^{i n}-\psi\right) \\
\dot{\zeta}=-\left(q_{1}+d\right) \zeta+q_{1} \eta+\beta\left(T_{s s}, T_{j}^{s s}\right)+\tilde{B}\left(\psi^{r}, \zeta\right) \\
\dot{\eta}=q_{2} \zeta-q_{2} \eta-q_{2}\left(T_{j}^{s s}-T_{s s}\right)+u_{s s}+\delta u
\end{array}\right.
$$

where $\beta\left(T_{s s}, T_{j}^{s s}\right)=q_{1}\left(T_{j}^{s s}-T_{s s}\right)+d\left(T^{i n}-T_{s s}\right), \tilde{r}\left(\psi^{r}, \zeta\right)=r\left(\psi^{r}+x_{s s}^{r}, \zeta+T_{s s}\right)$, $\psi^{i n}=x^{i n}-x_{s s} \in \mathbb{R}^{n}$ and the reaction heat

$$
\tilde{B}\left(\psi^{r}, \zeta\right)=B\left(\psi^{r}+x_{s s}^{r}, \zeta+T_{s s}\right)
$$

can be expressed as

$$
\tilde{B}\left(\psi^{r}, \zeta\right)=B_{0}+B_{1} \psi^{r}+B_{2} \zeta .
$$

In particular, if the kinetics follow the Arrhenius law, one has

$$
B_{0}=B_{0}\left(x_{s s}^{r}, T_{s s}\right)=H_{r}^{T} \tilde{r}_{0}\left(x_{s s}^{r}, T_{s s}\right)=H_{r}^{T}\left[\begin{array}{c}
\varphi_{1}\left(x_{1 s s}^{r}\right) k_{01} e^{-E_{1} / R T_{s s}} \\
\varphi_{2}\left(x_{2 s s}^{r}\right) k_{02} e^{-E_{2} / R T_{s s}} \\
\vdots \\
\varphi_{m}\left(x_{m s s}^{r}\right) k_{0 m} e^{-E_{m} / R T_{s s}}
\end{array}\right] \in \mathbb{R}
$$




$$
B_{1}=B_{1}\left(x^{r}, T\right)=H_{r}^{T}\left[\begin{array}{cccc}
B_{11} & 0 & \ldots & 0 \\
0 & B_{12} & \ldots & \vdots \\
\vdots & \vdots & \ldots & 0 \\
0 & 0 & \ldots & B_{1 m}
\end{array}\right] \in\left(\mathbb{R}^{m}\right)^{*}
$$

with

$$
\begin{gathered}
B_{11}=B_{11}\left(x_{1}^{r}, T\right)=\left(\frac{\varphi_{1}\left(x_{1}^{r}\right)-\varphi_{1}\left(x_{1 s s}^{r}\right)}{x_{1}^{r}-x_{1 s s}^{r}}\right) k_{01} e^{-E_{1} / R T} \in \mathbb{R} \\
\vdots \\
B_{1 m}=B_{1 m}\left(x_{m}^{r}, T\right)=\left(\frac{\varphi_{m}\left(x_{m}^{r}\right)-\varphi_{m}\left(x_{m s s}^{r}\right)}{x_{m}^{r}-x_{m s s}^{r}}\right) k_{0 m} e^{-E_{m} / R T} \in \mathbb{R}
\end{gathered}
$$

and

$$
B_{2}=B_{2}\left(x_{s s}^{r}, T\right)=H_{r}^{T}\left[\begin{array}{c}
\varphi_{1}\left(x_{1 s s}^{r}\right) k_{01}\left[\frac{e^{-E_{1} / R T}-e^{-E_{1} / R T_{s s}}}{T-T_{s s}}\right] \\
\varphi_{2}\left(x_{2 s s}^{r}\right) k_{02}\left[\frac{e^{-E_{2} / R T}-e^{-E_{2} / R T_{s s}}}{T-T_{s s}}\right] \\
\vdots \\
\varphi_{m}\left(x_{m s s}^{r}\right) k_{0 m}\left[\frac{e^{-E_{m} / R T}-e^{-E_{m} / R T_{s s}}}{T-T_{s s}}\right]
\end{array}\right] \in \mathbb{R} .
$$

The functions $\varphi_{i}\left(x_{i}^{r}\right)$ are the components of the diagonal matrix $\varphi\left(x^{r}\right)$ as in the reaction rate (2). It must be noted that, by definition of deviation coordinates, the system (11) takes the simplified form

$$
\left\{\begin{array}{l}
\dot{\psi}=C \tilde{r}\left(\psi^{r}, \zeta\right)+d\left(\psi^{i n}-\psi\right) \\
\dot{\zeta}=-\left(q_{1}+d\right) \zeta+q_{1} \eta+B_{1} \psi^{r}+B_{2} \zeta \\
\dot{\eta}=q_{2} \zeta-q_{2} \eta+\delta u
\end{array}\right.
$$

\section{Main Assumptions and Problem Statement}

Together with the assumptions $\boldsymbol{H p} \mathbf{1}$ ) to $\boldsymbol{H p} \boldsymbol{6}$ ), which are used to derive the mathematical description (8), further assumptions are now introduced to describe the dynamic behaviour of the system in particular operating conditions, to characterise the heat exchange of the reaction and to define the control bounds and the location of the measurement devices. A detailed analysis of the dynamic properties of system (8) may be found in previous works $[23,24,26,35,36]$. 
Assumption 1 For any initial value $x(0) \in \mathbb{R}_{+}^{n}, x(t) \in \mathbb{R}_{+}^{n}$ for all $t \geq 0$.

This assumption expresses the non-negativity property of the concentration variables.

Assumption 2 For the isothermal condition, $T=T_{s s}$, the system

$$
\dot{x}=C r\left(x^{r}, T_{s s}\right)+d\left(x^{i n}-x\right)
$$

has a single equilibrium point $x=x_{s s}$, which is globally exponentially stable.

This assumption states that the concentrations of the $n$ components of the reaction mixture reach their steady state values when the temperature is at its steady state value. Several industrial chemical reaction processes verify this assumption. The dynamics (18) can also be interpreted as the isothermal dynamics of the reactor (8). Moreover, by Lyapunov converse theorems [25], Assumption 2 may be reformulated as follows.

There exists a positive definite and proper function $W(\psi)$ such that

$$
\begin{gathered}
\alpha_{0} \phi^{2}(\psi) \leq W(\psi) \leq \alpha_{1} \phi^{2}(\psi) \\
\dot{W}=\left[\frac{\partial W}{\partial x}\right]^{T}\left[C r\left(x^{r}, T_{s s}\right)+d\left(x^{i n}-x\right)\right] \leq-\alpha_{2} \phi^{2}(\psi) \leq 0 \\
\left\|\frac{\partial W}{\partial x}\right\| \leq \alpha_{3} \phi(\psi)
\end{gathered}
$$

with $\alpha_{0}, \alpha_{1}, \alpha_{2}$ and $\alpha_{3}$ positive constants, and $\phi(\psi)=\|\psi\|$.

Assumption 3 The functions $k_{i}(T) \in \mathbb{R}^{m}$ satisfy the Lipschitz condition at $T=T_{\text {ss }}$, i.e. there exists a positive number $M$ such that the following relation holds

$$
\left\|C r\left(x^{r}, T\right)-C r\left(x^{r}, T_{s s}\right)\right\| \leq M\left|T-T_{s s}\right|
$$

for all $T$.

It must be noted that this assumption is satisfied by most kinetic models proposed in the literature (see $e . g .[18]$ ).

Assumption 4 The variables available for feedback are the dilution rate $d$, and the temperatures $T, T_{j}$ and $T^{\text {in }}$. 
As already observed, in industrial practice, concentration measurement devices are expensive, while temperatures and flowrates are simple to measure with devices that are accurate and reliable.

In what follows the system (8) and the Assumptions 1 to 4 are considered. The objective consists of finding, if possible, a static control law, described by equations of the form

$$
w=w(y)
$$

where $w^{T}=\left[u, d, T^{i n}\right]$ and $y^{T}=\left[T, T_{j}, T^{i n}, d\right]$, or a dynamics control law with state $\theta$, described by equations of the form

$$
\begin{aligned}
\dot{\theta} & =\Theta(\theta, y) \\
w & =w(\theta, y)
\end{aligned}
$$

such that the closed loop system (8)-(22) or (8)-(23) is globally asymptotically stable or locally asymptotically stable with a computable bound on the region of attraction. Moreover, the manipulated variables $w^{T}=\left[u, d, T^{i n}\right]$ take value inside a compact set $\mathcal{D} \subset \mathbb{R}^{3}$ which depends on the specific application.

\section{A Preliminary Result}

This section provides a simple result, which is instrumental in proving the main results of this work.

Lemma 1 Consider the system

$$
\dot{z}=f(z)+g(z) u
$$

with state $z \in \mathbb{R}^{n}$ and control $u \in \mathbb{R}$. Suppose that there exist an unknown constant control $u=u_{s s}$ and a positive definite and proper function $V(z)$ such that

$$
\dot{V}(z)=\frac{\partial V}{\partial z} f(z)+\frac{\partial V}{\partial z} g(z) u_{s s}<0
$$

for all nonzero $z$. Then, for any $\underline{u}$ and $\bar{u}$ such that $\underline{u} \leq u_{s s} \leq \bar{u}$, there exists a dynamic control law, which does not require knowledge of $u_{s s}$, namely

$$
\begin{aligned}
\dot{\theta} & =\Theta(z, \theta) \\
u & =\theta
\end{aligned}
$$

with $\theta(0) \in(\underline{u}, \bar{u})$, such that the closed loop system (24)-(26) is stable in the sense of Lyapunov, the variable $z$ converges to zero and, along the trajectories of the closed loop system, $\theta(t)=u(t) \in[\underline{u}, \bar{u}]$. 
Proof of Lemma 1 Consider the function

$$
V(z)+\omega(\theta)
$$

where

$$
\omega(\theta)=\ln (\bar{u}-\theta)^{\tau / k}-\ln (\theta-\underline{u})^{(\tau+1) / k}
$$

with $k>0, \tau=\frac{u_{s s}-\bar{u}}{\bar{u}-\underline{u}}<0$ and $\tau+1>0$. Note that $V(z)+\omega(\theta)$ is positive definite and proper in $\bar{\Omega}_{z \theta}=\left\{(z, \theta) \in \mathbb{R}^{(n+1)} \mid \underline{u}<\theta<\bar{u}\right\}$. Then, differentiation with respect to time gives

$$
\begin{aligned}
\dot{V}(z)+\dot{\omega}(\theta) & =\frac{\partial V}{\partial z}[f(z)+g(z) \theta]+\frac{\partial \omega}{\partial \theta} \dot{\theta} \\
& =\frac{\partial V}{\partial z}\left[f(z)+g(z) u_{s s}\right]+\frac{\partial V}{\partial z} g(z)\left(\theta-u_{s s}\right)+\frac{\partial \omega}{\partial \theta} \dot{\theta} .
\end{aligned}
$$

Setting

$$
\dot{\theta}=\frac{\partial V}{\partial z} g(z) \sigma(\theta)
$$

with

$$
\sigma(\theta)=k(\theta-\bar{u})(\theta-\underline{u})
$$

and noting that

$$
\theta-u_{s s}+\frac{\partial \omega}{\partial \theta} \sigma(\theta)=0
$$

yields

$$
\begin{aligned}
\dot{V}(z)+\dot{\omega}(\theta) & =\frac{\partial V}{\partial z}\left[f(z)+g(z) u_{s s}\right]+\frac{\partial V}{\partial z} g(z)\left[\theta-u_{s s}+\frac{\partial \omega}{\partial \theta} \sigma(\theta)\right] \\
& =\frac{\partial V}{\partial z}\left[f(z)+g(z) u_{s s}\right]<0
\end{aligned}
$$

for all nonzero $z$. As a result the closed loop system

$$
\begin{aligned}
\dot{z} & =f(z)+g(z) u \\
\dot{\theta} & =\Theta(z, \theta) \\
u & =\theta
\end{aligned}
$$

is locally stable and any trajectory starting in the set $\Omega_{z \theta}$ is such that the variable $z$ converges to zero and $\theta(t)=u(t) \in[\underline{u}, \bar{u}]$ for all $t$.

From equation (30) it is easy to show that, if

$$
y=\frac{\partial V}{\partial z} g(z)
$$


is the measurable variable, then the dynamic control law (30) may be interpreted as a nonlinear PI controller given by

$$
u=\frac{\bar{u}[u(0)-\underline{u}]-\underline{u}[u(0)-\bar{u}] \phi_{n l}(y)}{u(0)-\underline{u}-[u(0)-\bar{u}] \phi_{n l}(y)}
$$

where $\phi_{n l}(y)=\exp \left[k(\bar{u}-\underline{u}) \int_{0}^{t_{f}} y(t) d t\right]$ and $u(0)$ is the value of the control input at the initial time.

Remark 1 Lemma 1 can be clarified as follows. If the system (24) in closed loop with the constant control $u=u_{s s}$ is globally asymptotically stable, then it is possible to design a dynamic control law, which does not require precise knowledge of the steady state control $u_{s s}$, yielding a closed loop system which is locally stable and such that $\lim _{t \rightarrow \infty} z(t)=0$. In general, this dynamic control law requires knowledge of the state of the system. However, in the next section, it will be shown that for the special case of chemical reactors the control law requires only partial state measurement.

Remark 2 The dynamic control law constructed in the proof of Lemma 1 requires knowledge of an upper and lower bound on the steady state (stabilising) input $u_{s s}$ and provides a bounded control action, i.e. the control variable is (uniformly) bounded for all $t$. This is particularly important in chemical engineering applications.

Remark 3 The hypothesis that the system (24) in closed loop with the constant control $u=u_{s s}$ is globally asymptotically stable can be experimentally verified and this kind of information is in general available in real applications, although the precise value of the constant stabilising $u_{s s}$ is not known or might depend on the operating conditions.

\section{Main Results}

In this section it is shown how the problem of asymptotic stabilisation for system (24) can be solved using dynamic control laws which require only partial state information and provide a bounded control action.

The result established in Lemma 1 will be instrumental in deriving the control laws. However, as already discussed, the application of Lemma 1 requires the property that the system can be stabilised with constant control action. This 
property is closely related to the features of the temperature sub-system and, in particular, to the degree of exothermicity of the reaction taking place in the reactor. These are in turn related to the properties of the mappings $B_{1}$ and $B_{2}$ given in equations (14) and (16).

It must be noted that a CSTR can be controlled using different variables. To begin with, the heat supplied or removed from the jacket, $u$, is considered to be the manipulated variable and the jacket temperature $T_{j}$ is assumed to be the measured variable. Then, multivariable extensions, in which the manipulated variables are the pair $\left(u, T^{i n}\right)$ or $(u, d)$ and the measured variables are $\left(T, T_{j}, d\right)$, are considered. In all cases the control laws provide bounded control action and, in particular, the positivity constraints associated with some variables, e.g. the dilution rate $d$, will be satisfied for all time.

Remark 4 The results described in this section are mainly global, i.e. under certain assumptions, the closed loop system is globally asymptotically stable in its domain of definition. The only exception is the case of the manipulated variables $\left(u, T^{i n}\right)$ and the measured variables $\left(T, T_{j}\right)$ where only local stability, with an estimation of the region of attraction, is proved. This is because, as detailed in the proof of Proposition 2, the positivity constraints on the manipulated variable $T^{\text {in }}$ cannot be satisfied in the whole state space.

Remark 5 It will be apparent from the forthcoming discussion that the condition needed to guarantee global or local asymptotic stability of the closed loop system depend upon the control pattern. In particular, the assumption required in the case of the single manipulated variable $u$ can be substantially weakened if other control variables, such as $T^{\text {in }}$ or $d$, are employed.

\subsection{Stabilisation Through Control of the Supplied or Removed Heat $u$}

Proposition 1 Consider the CSTR described by equation (11) and suppose that Assumptions 1 to 4 hold. Consider the reaction heat $\tilde{B}\left(\psi^{r}, \zeta\right)$ expressed by equation (12), i.e.

$$
\tilde{B}\left(\psi^{r}, \zeta\right)=B_{0}+B_{1} \psi^{r}+B_{2} \zeta
$$

and suppose that the functions $B_{1}$ and $B_{2}$ defined in (14) and (16) are such that

$$
\bar{B}_{2}+\frac{\alpha_{3} M}{\alpha_{2}} \bar{\Omega}<d
$$


where $\bar{B}_{2}=\max _{\forall T \in \mathbb{R}_{+}} B_{2}, M$ is as in (21), $d$ is the dilution rate, $\alpha_{2}$ and $\alpha_{3}$ are as in (19) and (20) respectively, and $\bar{\Omega}=\max _{\forall x^{r}, T}\left\|\left(P^{r}\right)^{T} B_{1}^{T}\right\|$ with $P^{r}$ as in (10).

Then there exist a constant feedback law

$$
u=u_{s s},
$$

two real-valued functions, $W(\psi)$ and $V(\zeta, \eta)$, which are positive definite and proper, and a real positive constant $\lambda^{*}$ such that

$$
\dot{W}+\lambda^{*} \dot{V}<0
$$

for all $(\psi, \zeta, \eta) \neq(0,0,0)$. Therefore, the constant control (35) globally asymptotically stabilises the system (11) at the equilibrium point $(0,0,0)$.

Remark 6 Before presenting the proof of Proposition 1, it is convenient to briefly discuss condition (34). This condition implies that $\bar{B}_{2}<d$ which is always verified if the reaction network is globally endothermic (in fact, for endothermic reactions $B_{2}<0$ for all $T \in \mathbb{R}_{+}$). By a simple property of any projection operator, condition (34) can be simplified as

$$
\max _{\forall x^{r}, T}\left\|B_{1}^{T}\right\|<\frac{\alpha_{2}}{\alpha_{3} M}\left(d-\bar{B}_{2}\right) \text {. }
$$

Moreover, $\left\|B_{1}^{T}\right\|$ and $B_{2}$ are bounded functions of their arguments and this assumption can be satisfied if the dilution rate $d$ is sufficiently large, which is in agreement with common process industry experience. It is also worth noting that the functions $B_{1}$ and $B_{2}$ and the variables $M, \alpha_{2}$ and $\alpha_{3}$ can be, in practical applications, experimentally estimated.

Proof of Proposition 1 Let $W(\psi)$ be as in Assumption 2 and let $V(\zeta, \eta)$ be defined as

$$
V(\zeta, \eta)=\frac{q_{2}}{2}\left(T-T_{s s}\right)^{2}+\frac{q_{1}}{2}\left(T_{j}-T_{j}^{s s}\right)^{2}=\frac{q_{2}}{2} \zeta^{2}+\frac{q_{1}}{2} \eta^{2} .
$$

Then

$$
\begin{aligned}
\dot{W} & =\left[\frac{\partial W}{\partial x}\right]^{T}\left[C r\left(x^{r}, T\right)+d\left(x^{i n}-x\right)\right] \\
& =\left[\frac{\partial W}{\partial x}\right]^{T}\left[\operatorname{Cr}\left(x^{r}, T^{s s}\right)+d\left(x^{i n}-x\right)\right]+\left[\frac{\partial W}{\partial x}\right]^{T}\left[\operatorname{Cr}\left(x^{r}, T\right)-\operatorname{Cr}\left(x^{r}, T^{s s}\right)\right] \\
& \leq-\alpha_{2} \phi^{2}+\left\|\frac{\partial W}{\partial x}\right\|\left\|C r\left(x^{r}, T\right)-C r\left(x^{r}, T^{s s}\right)\right\| \\
& \leq-\alpha_{2} \phi^{2}+\alpha_{3} \phi M\left|T-T_{s s}\right|=-\alpha_{2}\|\psi\|^{2}+\alpha_{3}\|\psi\| M|\zeta|
\end{aligned}
$$


and, for $u=u_{s s}$,

$$
\dot{V}=q_{2} \zeta \dot{\zeta}+q_{1} \eta \dot{\eta}=-q_{2}\left(q_{1}+d-B_{2}\right) \zeta^{2}-q_{1} q_{2} \eta^{2}+2 q_{1} q_{2} \zeta \eta+q_{2} B_{1} \psi^{r} \zeta
$$

where $\dot{\zeta}$ and $\dot{\eta}$ are as in equation (17). Suppose now that condition (34) is satisfied. Then, for any $\lambda>0$

$$
\begin{aligned}
\dot{W}+\lambda \dot{V} \leq & -\alpha_{2}\|\psi\|^{2}+\alpha_{3}\|\psi\| M|\zeta|+\lambda q_{2} B_{1} \psi^{r} \zeta-\lambda q_{2}\left(q_{1}+d-B_{2}\right) \zeta^{2} \\
& -\lambda q_{1} q_{2} \eta^{2}+2 \lambda q_{1} q_{2} \zeta \eta \\
= & -\frac{\alpha_{2}}{2}\|\psi\|^{2}+\alpha_{3} M\|\psi\||\zeta|-\frac{\alpha_{2}}{2}\|\psi\|^{2}+\lambda q_{2} B_{1} P^{r} \psi \zeta \\
& -\lambda q_{2}\left(q_{1}+d-B_{2}\right) \zeta^{2}-\lambda q_{1} q_{2} \eta^{2}+2 \lambda q_{1} q_{2} \zeta \eta
\end{aligned}
$$

and, after simple manipulation, one has

$$
\begin{aligned}
\dot{W}+\lambda \dot{V} \leq & -\left[\sqrt{\frac{\alpha_{2}}{2}}\|\psi\|-\frac{\alpha_{3} M}{2 \sqrt{\frac{\alpha_{2}}{2}}}|\zeta|^{2}-\left\|\sqrt{\frac{\alpha_{2}}{2}} \psi-\frac{\lambda q_{2}\left(B_{1} P^{r}\right)^{T}}{2 \sqrt{\frac{\alpha_{2}}{2}}} \zeta\right\|^{2}\right. \\
& -\left[\sqrt{\lambda q_{1} q_{2}} \eta-\sqrt{\lambda q_{1} q_{2}} \zeta\right]^{2} \\
& -\left[\lambda q_{2}\left(d-B_{2}\right)-\frac{\alpha_{3}^{2} M^{2}}{2 \alpha_{2}}-\frac{\lambda^{2} q_{2}^{2}\left\|\left(P^{r}\right)^{T} B_{1}^{T}\right\|^{2}}{2 \alpha_{2}}\right] \zeta^{2} .
\end{aligned}
$$

The first three terms of equation (40) are non-positive for any non-zero value of the variables $\psi, \zeta$ and $\eta$, while the sign of the fourth term is unknown and depends on the choice of $\lambda$. However, this last term can be made negative if there exists a $\lambda>0$ such that

$$
\lambda q_{2}\left(d-B_{2}\right)-\frac{\alpha_{3}^{2} M^{2}}{2 \alpha_{2}}-\frac{\lambda^{2} q_{2}^{2}\left\|\left(P^{r}\right)^{T} B_{1}^{T}\right\|^{2}}{2 \alpha_{2}}>0
$$

or equivalently

$$
q_{2}^{2}\left\|\left(P^{r}\right)^{T} B_{1}^{T}\right\|^{2} \lambda^{2}-2 q_{2} \alpha_{2}\left(d-B_{2}\right) \lambda+\alpha_{3}^{2} M^{2}<0
$$

Such a $\lambda$ indeed exists and it is given by

$$
\lambda^{*}=\frac{\alpha_{2}\left(d-\bar{B}_{2}\right)}{q_{2}\left(\max _{\forall x^{r}, T}\left\|\left(P^{r}\right)^{T} B_{1}^{T}\right\|\right)^{2}}>0 .
$$

In fact, by condition (34), one has

$$
q_{2}^{2}\left\|\left(P^{r}\right)^{T} B_{1}^{T}\right\|^{2}\left(\lambda^{*}\right)^{2}-2 q_{2} \alpha_{2}\left(d-B_{2}\right) \lambda^{*}+\alpha_{3}^{2} M^{2}<0 .
$$


It is straightforward to conclude that the function

$$
W(\psi)+\lambda^{*} V(\zeta, \eta)
$$

is a Lyapunov function for the system (11) with $u=u_{s s}$, hence the equilibrium point $(0,0,0)$ of the closed loop system (11)-(35) is GAS.

Proposition 1 can be combined with the result established in Lemma 1 as expressed in the following corollary, in which, instead of the constant control $u=u_{s s}$, a dynamic control law, which requires only knowledge of the jacket temperature and of its set-point value, is used.

Corollary 1 Consider the CSTR described in equation (11) and suppose that Assumptions 1 to 4 hold and that condition (34) is verified. Assume moreover that $u$ is the only manipulated variable and that $u \in(\underline{u}, \bar{u})$ and $u_{s s} \in[\underline{u}, \bar{u}]$.

Then the initialized dynamic output feedback control law

$$
\left\{\begin{array}{l}
u=\theta \\
\dot{\theta}=k_{\theta}(\theta-\bar{u})(\theta-\underline{u}) \eta=k_{\theta}(\theta-\bar{u})(\theta-\underline{u})\left(T_{j}-T_{j}^{s s}\right)
\end{array}\right.
$$

with $k_{\theta}>0$, is such that the equilibrium $\left(0,0,0, u_{s s}\right)$ of the closed loop system (11)-(45) is locally stable and, for any initial conditions $(\psi(0), \zeta(0), \eta(0), \theta(0))$ with $\theta(0) \in(\underline{u}, \bar{u}),(\psi(t), \zeta(t), \eta(t))$ converges asymptotically to $(0,0,0)$ and the control variable $\theta(t) \in(\underline{u}, \bar{u})$.

Proof of Corollary 1 The proof is a simple consequence of the results proved in Lemma 1 and Proposition 1. Simple manipulations show that the positive definite and proper function

$$
W(\psi)+\lambda^{*} V(\zeta, \eta)+\omega(\theta)
$$

where $W(\psi)$ and $V(\zeta, \eta)$ are as in the proof of Proposition 1,

$$
\omega(\theta)=\lambda^{*} q_{1} \ln (\bar{u}-\theta)^{\tau / k_{\theta}}-\lambda^{*} q_{1} \ln (\theta-\underline{u})^{(\tau+1) / k_{\theta}}
$$

with

is such that

$$
\tau=\frac{u_{s s}-\bar{u}}{\bar{u}-\underline{u}}<0
$$

$$
\begin{aligned}
\dot{W}+\lambda^{*} \dot{V}+\dot{\omega} \leq & -\alpha_{2}\|\psi\|^{2}+\alpha_{3} M\|\psi\||\zeta|+\lambda q_{2} B_{1} P^{r} \psi \zeta-\lambda q_{2}\left(q_{1}+d-B_{2}\right) \zeta^{2} \\
& +2 \lambda q_{1} q_{2} \zeta \eta-\lambda q_{1} q_{2} \eta^{2}
\end{aligned}
$$

which is non-positive as shown in the proof of Proposition 1, hence the claim. $\triangleleft$ 
The above corollary shows that, for the reactor (8), temperature regulation can be achieved by a bounded control action without any knowledge of the steady state control input $u_{s s}$, but only with the knowledge of the set-point jacket temperature $T_{j}^{s s}$. This set-point value is in general simpler to compute via experiments than $u_{s s}$.

The stabilising control has a very simple structure, however it requires that condition (34), concerning the level of exothermicity of the reaction network, is satisfied. This condition, which might be too restrictive, can be relaxed increasing the complexity of the control structure, i.e. introducing extra manipulated variables to compensate the physical limitations of the heat exchange when a highly exothermic reaction takes place, as discussed hereafter.

\subsection{Stabilisation Through Control of the Supplied or Removed Heat $u$ and of the Inlet Stream Temperature $T^{\text {in }}$}

In this section, the case in which the control variables are the heat in put rate, $u$, and the reactor inlet temperature, $T^{\text {in }}$, is studied and it is shown how condition (34) is accordingly relaxed. Consider system (8) and, in addition to the variables (9), introduce the new deviation variable

$$
\delta T^{i n}=T^{i n}-T_{s s}^{i n} \in \mathbb{R} .
$$

As a result, system (8) can be rewritten as

$$
\left\{\begin{aligned}
\dot{\psi} & =C \tilde{r}(\psi, \zeta)+d\left(\psi^{i n}-\psi\right) \\
\dot{\zeta} & =-\left(q_{1}+d\right) \zeta+q_{1} \eta+d \delta T^{i n}+B_{1} \psi^{r}+B_{2} \zeta \\
\dot{\eta} & =-q_{2} \eta+q_{2} \zeta+\delta u
\end{aligned}\right.
$$

which is the starting point in deriving a multivariable version of Proposition 1.

Proposition 2 Consider the CSTR described by equations (49) and suppose that Assumptions 1 to 4 hold. Consider the reaction heat $\tilde{B}\left(\psi^{r}, \zeta\right)$, defined in (12), and suppose that for a given $k_{\text {in }} \geq 0$,

$$
\bar{B}_{2}+\frac{\alpha_{3} M}{\alpha_{2}} \bar{\Omega}<d+k_{i n} d
$$

where $B_{2}$ is as in (16), $\bar{B}_{2}=\max _{\forall T \in \mathbb{R}_{+}} B_{2}, M$ is as in (21), $d$ is the dilution rate, $\alpha_{2}$ and $\alpha_{3}$ are as in (19) and (20) respectively, $\bar{\Omega}=\max _{\forall x^{r}, T}\left\|\left(P^{r}\right)^{T} B_{1}^{T}\right\|$, with $B_{1}$ as in (14). 
Then there exists two positive constants $u_{s s}$ and $T_{s s}^{i n}$ such that the equilibrium $(0,0,0)$ of the system (49) in closed loop with the feedback law

$$
\begin{aligned}
u & =u_{s s} \\
T^{i n} & =T_{s s}^{i n}-k_{i n}\left(T-T_{s s}\right)
\end{aligned}
$$

is globally asymptotically stable. Moreover, if

$$
0 \leq k_{i n} \leq \frac{T_{s s}^{i n}}{\bar{\psi}} \frac{\alpha_{3}}{\alpha_{2}} M \sqrt{\frac{q_{2}}{q_{1}+q_{2}}}
$$

with $\bar{\psi}=\max \|\psi\|$, then all trajectories of the closed loop system (49)-(51) such that $T(0)$ and $T_{j}(0)$ are in the set

$$
\mathcal{H}=\left\{\left(T, T_{j}\right) \mid \frac{q_{2}}{2}\left(T-T_{s s}\right)^{2}+\frac{q_{1}}{2}\left(T_{j}-T_{j}^{s s}\right)^{2} \leq \frac{q_{2}}{2}\left(\frac{T_{s s}^{i n}}{k_{i n}}\right)^{2}\right\}
$$

remain in the set $\mathcal{H}$, and are such that

$$
T^{i n}=T_{s s}^{i n}-k_{i n}\left(T-T_{s s}\right)
$$

is non-negative for all $t \geq 0$.

Proof of Proposition 2 We break up the proof in two steps.

Step 1: Global asymptotic stability. Consider the positive definite and proper function

$$
W(\psi)+\lambda V(\zeta, \eta)
$$

where $W(\psi)$ and $V(\zeta, \eta)$ are as in the proof of Proposition 1 and $\lambda$ is a positive constant to be determined. Then, along the trajectories of the closed loop system (49)-(51) one has

$$
\begin{aligned}
\dot{W}+\lambda \dot{V} \leq & -\alpha_{2} \phi^{2}+\alpha_{3} M\|\psi\||\zeta|+\lambda q_{2} B_{1} \psi^{r} \zeta-\lambda q_{2}\left(q_{1}+d-B_{2}\right) \zeta^{2} \\
& +\lambda q_{2} d \zeta \delta T^{i n}-\lambda q_{1} q_{2} \eta^{2}+2 \lambda q_{1} q_{2} \zeta \eta \\
= & -\frac{\alpha_{2}}{2}\|\psi\|^{2}+\alpha_{3} M\|\psi\||\zeta|-\frac{\alpha_{2}}{2}\|\psi\|^{2}+\lambda q_{2} B_{1} P^{r} \psi \zeta \\
& -\lambda q_{2}\left(q_{1}+d+k_{i n} d-B_{2}\right) \zeta^{2}-\lambda q_{1} q_{2} \eta^{2}+2 \lambda q_{1} q_{2} \zeta \eta
\end{aligned}
$$


and, after simple manipulation,

$$
\begin{aligned}
& \dot{W}+\lambda \dot{V} \leq-\left[\sqrt{\frac{\alpha_{2}}{2}}\|\psi\|-\frac{\alpha_{3} M}{2 \sqrt{\frac{\alpha_{2}}{2}}}|\zeta|\right]^{2}-\left\|\sqrt{\frac{\alpha_{2}}{2}} \psi-\frac{\lambda q_{2}\left(B_{1} P^{r}\right)^{T}}{2 \sqrt{\frac{\alpha_{2}}{2}}} \zeta\right\|^{2} \\
& -\left[\sqrt{\lambda q_{1} q_{2}} \eta-\sqrt{\lambda q_{1} q_{2}} \zeta\right]^{2} \\
& -\left[\lambda q_{2}\left(d+k_{i n} d-B_{2}\right)-\frac{\alpha_{3}^{2} M^{2}}{2 \alpha_{2}}-\frac{\lambda^{2} q_{2}^{2}\left\|\left(P^{r}\right)^{T} B_{1}^{T}\right\|^{2}}{2 \alpha_{2}}\right] \zeta^{2} .
\end{aligned}
$$

Applying arguments similar to those used in the proof of Proposition 1, it is easy to conclude that there exists a positive constant $\lambda^{*}$ such that

$$
\dot{W}+\lambda^{*} \dot{V}<0
$$

for all $(\psi, \zeta, \eta) \neq(0,0,0)$, hence the claim.

Step 2: Invariance of the set $\mathcal{H}$. Note that the control $T^{\text {in }}$ defined in (51) is non-negative for all

$$
T \leq T_{s s}+\frac{T_{s s}^{i n}}{k_{i n}}
$$

Hence, a simple calculation shows that it is non-negative within the set $\mathcal{H}$ (see also Figure 14 for a graphical interpretation). Therefore, to establish the claim, it must be proved that the set $\mathcal{H}$ is positively invariant. To this end, consider the function $V$ in Step 1, and note that

$$
\begin{aligned}
\dot{V} \leq & -q_{1} q_{2}\left[\left(T-T_{s s}\right)-\left(T_{j}-T_{j}^{s s}\right)\right]^{2}-q_{2}\left(d+d k_{i n}-\bar{B}_{2}\right)\left(T-T_{s s}\right)^{2} \\
& +q_{2}\left(T-T_{s s}\right) \frac{\alpha_{2}}{\alpha_{3} M}\left(d+d k_{i n}-\bar{B}_{2}\right) \bar{\psi}
\end{aligned}
$$

If $k_{i n}$ satisfies condition (52) then, as shown in Appendix A, the function $\dot{V}$ is positive semidefinite in a compact set properly contained in $\mathcal{H}$, and negative elsewhere. Hence the claim.

The control law (51) cannot be implemented because it requires knowledge of the steady state variables $u_{s s}$ and $T_{s s}^{i n}$. However, as already discussed, this problem can be easily overcome, as stated in the following corollary, the proof of which is similar to the proof of Corollary 1 , hence it is omitted.

Corollary 2 Consider the CSTR described in (49) and suppose that Assumptions 1 to 4 hold. Suppose that condition (50) is satisfied. Assume that $u$ and 
$T^{i n}$ are the manipulated variables, that $u \in(\underline{u}, \bar{u})$ and $T^{i n} \in\left(\underline{T}^{i n}, \bar{T}^{i n}\right)$, and that $u_{s s} \in(\underline{u}, \bar{u})$ and $T_{s s}^{i n} \in\left(\underline{T}^{i n}, \bar{T}^{i n}\right)$.

Then the initialized dynamic output feedback control law

$$
\left\{\begin{aligned}
u & =\theta \\
\dot{\theta} & =k_{\theta}(\theta-\underline{u})(\theta-\bar{u}) \eta=k_{\theta}(\theta-\underline{u})(\theta-\bar{u})\left(T_{j}-T_{j}^{s s}\right) \\
T^{i n} & =\gamma-k_{i n}\left(T-T_{s s}\right) \\
\dot{\gamma} & =k_{\gamma}\left(\gamma-\underline{T}^{i n}\right)\left(\gamma-\bar{T}^{i n}\right) \zeta=k_{\gamma}\left(\gamma-\underline{T}^{i n}\right)\left(\gamma-\bar{T}^{i n}\right)\left(T-T_{s s}\right)
\end{aligned}\right.
$$

with $k_{\theta}>0, k_{\text {in }} \geq 0$ and $k_{\gamma}>0$, is such that the equilibrium point $\left(0,0,0, u_{s s}, T_{s s}^{\text {in }}\right)$ of the closed loop (49)-(58) is locally stable and, for any initial conditions $(\psi(0), \zeta(0), \eta(0), \theta(0), \gamma(0))$ with $\theta(0) \in(\underline{u}, \bar{u})$ and $\gamma(0) \in\left(\underline{T}^{i n}, \bar{T}^{i n}\right),(\psi(t), \zeta(t), \eta(t))$ converges asymptotically to $(0,0,0)$ and the control variables are such that $\theta(t) \in(\underline{u}, \bar{u})$ and $\gamma(t) \in\left(\underline{T}^{i n}, \bar{T}^{i n}\right)$.

Moreover, if

$$
k_{i n} \leq \frac{T_{s s}^{i n}}{\bar{\psi}} \frac{\alpha_{3}}{\alpha_{2}} M \sqrt{\frac{q_{1}}{q_{1}+q_{2}}}
$$

then every trajectory of the closed loop system starting in $\mathcal{H}$ remains in $\mathcal{H}$ and $T^{\text {in }} \geq 0$ for all $t \geq 0$.

\subsection{Stabilisation through Control of the Supplied or Removed Heat $u$ and of the Dilution Rate $d$}

A runaway instability can occur when the heat produced by exothermic reactions cannot be removed by heat exchange with the cooling jacket. Heat is accumulated inside the reactor because the heat exchanger is not capable of removing the overheating due to physical limitations. If heat cannot be added or removed fast enough from the CSTR, then the process volume must be decreased until heat transfer occurs at the proper rate, or, for an exothermic reaction, the rate of product formation must be reduced. Thus it is necessary to consider an additional action in order to cool the reactor. A feasible possibility is to decrease the dilution rate $d$, namely the quantity of reactant fed to the reactor in order to reduce the reaction rates, hence the heat produced.

Consider the reactor system described by (8), let the dilution rate, $d$, be a new control input, which is acting together with the manipulated transferred 
heat $u$, and introduce the new deviation variable

$$
\delta d=d-d_{s s} \in \mathbb{R}
$$

As a result, the system (8) becomes

$$
\left\{\begin{aligned}
\dot{\psi} & =C \tilde{r}\left(\psi^{r}, \zeta\right)+d\left(\psi^{i n}-\psi\right) \\
\dot{\zeta} & =-\left(q_{1}+d_{s s}+\delta d-B_{2}\right) \zeta+q_{1} \eta+\delta d\left(T^{i n}-T_{s s}\right)+B_{1} P^{r} \psi \\
\dot{\eta} & =-q_{2} \eta+q_{2} \zeta+\delta u
\end{aligned}\right.
$$

In the following proposition it is shown how this system can be stabilised at the desired equilibrium and how condition (34) is modified by the introduction of a further control input, namely the dilution rate $d$.

Proposition 3 Consider the CSTR described in (60) and suppose that Assumptions 1 to 4 hold. Consider the reaction heat $\tilde{B}\left(\psi^{r}, \zeta\right)$ as in (12), i.e.

$$
\tilde{B}\left(\psi^{r}, \zeta\right)=B_{0}+B_{1} \psi^{r}+B_{2} \zeta
$$

Suppose that

$$
B_{2}\left(T^{i n}\right)+\frac{\alpha_{3} M}{\alpha_{2}} \bar{\Omega}^{i n} \leq d_{s s}
$$

where $B_{2}\left(T^{i n}\right)$ is as in (16) with $T=T^{i n}, d$ is the dilution rate, $\alpha_{2}$ is as in (19), $\alpha_{3}$ is as in (20), $M$ is as in (21), $\bar{\Omega}^{i n}=\max _{\forall x^{r}}\left\|\left(P^{r}\right)^{T} B_{1}^{T}\left(T^{i n}\right)\right\|$ and $B_{1}\left(T^{i n}\right)$ is as in (14) with $T=T^{\text {in }}$. Then there exist a feedback control law

$$
\begin{aligned}
u & =u_{s s} \\
d & =d_{s s}+k_{d}\left[\operatorname{sat}\left(T-T_{s s}\right) \operatorname{sat}\left(T-T^{i n}\right)\right]
\end{aligned}
$$

with $k_{d}=\min \left(d_{s s}-\underline{d}, \bar{d}-d_{s s}\right)$ and sat the saturation function, two real-valued functions, $W(\psi)$ and $V(\zeta, \eta)$, which are positive definite and proper, and a real constant $\lambda^{*}$ such that

$$
\dot{W}+\lambda^{*} \dot{V}<0
$$

for all $(\psi, \zeta, \eta) \neq(0,0,0)$. Therefore, the control law (63) globally asymptotically stabilises the system (60) at the equilibrium point $(0,0,0)$.

Proof of Proposition 3 The proof is similar to the Proof of Proposition 1. Consider the positive definite and proper function

$$
W(\psi)+\lambda V(\zeta, \eta)
$$


where $W(\psi)$ and $V(\zeta, \eta)$ are as in Assumption 2 and equation (36) respectively. Differentiating the function (64) with respect to time yields

$$
\begin{aligned}
\dot{W}+\lambda \dot{V} \leq & -\frac{\alpha_{2}}{2}\|\psi\|^{2}+\alpha_{3} M\|\psi\||\zeta|-\frac{\alpha_{2}}{2}\|\psi\|^{2}+\lambda q_{2} B_{1} P^{r} \psi \zeta \\
& -\lambda q_{2}\left(q_{1}+d_{s s}-B_{2}\right) \zeta^{2}-\lambda q_{1} q_{2} \eta^{2}+2 \lambda q_{1} q_{2} \zeta \eta+\lambda q_{2}\left(T^{i n}-T\right) \delta d \zeta
\end{aligned}
$$

and, after some manipulation, one has

$$
\begin{aligned}
& \dot{W}+\lambda \dot{V} \leq-\left[\sqrt{\frac{\alpha_{2}}{2}}\|\psi\|-\frac{\alpha_{3} M}{2 \sqrt{\frac{\alpha_{2}}{2}}}|\zeta|\right]^{2}-\left\|\sqrt{\frac{\alpha_{2}}{2}} \psi-\frac{\lambda q_{2}\left(B_{1} P^{r}\right)^{T}}{2 \sqrt{\frac{\alpha_{2}}{2}}} \zeta\right\|^{2} \\
& -\left[\sqrt{\lambda q_{1} q_{2}} \eta-\sqrt{\lambda q_{1} q_{2}} \zeta\right]^{2} \\
& -\left[\lambda q_{2}\left(d_{s s}+\frac{\left(T-T^{i n}\right)}{\left(T-T_{s s}\right)} \delta d-B_{2}\right)-\frac{\alpha_{3}^{2} M^{2}}{2 \alpha_{2}}-\frac{\lambda^{2} q_{2}^{2}\left\|\left(P^{r}\right)^{T} B_{1}^{T}\right\|^{2}}{2 \alpha_{2}}\right] \zeta^{2} .
\end{aligned}
$$

It is easy to check that equation (66) is non-positive if

$$
\lambda q_{2}\left(d_{s s}+\frac{\left(T-T^{i n}\right)}{\left(T-T^{*}\right)} \delta d-B_{2}\right)-\frac{\alpha_{3}^{2} M^{2}}{2 \alpha_{2}}-\frac{\lambda^{2} q_{2}^{2}\left\|\left(P^{r}\right)^{T} B_{1}^{T}\right\|^{2}}{2 \alpha_{2}}>0
$$

and, in particular, if condition (67) is true for $T=T^{\text {in }}$, i.e.

$$
\lambda q_{2}\left(d_{s s}-B_{2}\left(T^{i n}\right)\right)-\frac{\alpha_{3}^{2} M^{2}}{2 \alpha_{2}}-\frac{\lambda^{2} q_{2}^{2}\left\|\left(P^{r}\right)^{T} B_{1}^{T}\left(T^{i n}\right)\right\|^{2}}{2 \alpha_{2}}>0,
$$

which is positive for $\lambda^{*}=\frac{\alpha_{2}\left(d_{s s}-B_{2}\left(T^{i n}\right)\right)}{q_{2}\left(\max _{\forall x^{r}}\left\|\left(P^{r}\right)^{T} B_{1}^{T}\left(T^{i n}\right)\right\|\right)^{2}}>0$.

Remark 7 It must be noted that condition (62) differs from condition (34), as it imposes a constraint on the exothermicity of the reaction only for $T=T^{\text {in }}$ and not for all temperatures $T$.

Obviously, even in this case, the control law (63) can be modified to avoid the use of the steady state variables $u_{s s}$ and $d_{s s}$.

Corollary 3 Consider the CSTR described in equations (49), suppose that Assumptions 1 to 4 hold and that condition (62) is verified. Assume moreover that $u$ and $d$ are the manipulated variables, that $u \in(\underline{u}, \bar{u})$ and $d \in(\underline{d}, \bar{d})$, and that $u_{s s} \in[\underline{u}, \bar{u}]$ and $d_{s s} \in[\underline{d}, \bar{d}]$. 
Then the initialized dynamic output feedback control law

$$
\left\{\begin{aligned}
u & =\theta \\
\dot{\theta} & =k_{\theta}(\theta-\underline{u})(\theta-\bar{u}) \eta=k_{\theta}(\theta-\underline{u})(\theta-\bar{u})\left(T_{j}-T_{j}^{s s}\right) \\
d & =v+k_{d}\left[\operatorname{sat}\left(T-T_{s s}\right) \operatorname{sat}\left(T-T^{i n}\right)\right] \\
\dot{v} & =k_{v}\left(T^{i n}-T_{s s}\right)(v-\underline{d})(v-\bar{d}) \zeta=k_{v}\left(T^{i n}-T_{s s}\right)(v-\underline{d})(v-\bar{d})\left(T-T_{s s}\right)
\end{aligned}\right.
$$

with $k_{\theta}>0, k_{d}>0$ and $k_{v}>0$, is such that the equilibrium $\left(0,0,0, u_{s s}, d_{s s}\right)$ of the closed loop system (60)-(68) is locally stable and, for any initial conditions $(\psi(0), \zeta(0), \eta(0), \theta(0), v(0))$ with $\theta(0) \in(\underline{u}, \bar{u})$ and $v(0) \in(\underline{d}, \bar{d}),(\psi(t), \zeta(t), \eta(t))$ converges asymptotically to $(0,0,0)$ and the control variables are such that $\theta(t) \in$ $(\underline{u}, \bar{u})$ and $v(t) \in(\underline{d}, \bar{d})$.

\section{Case Studies}

The control methodology developed so far is applied to stabilise the temperature in two chemical reactors. It is assumed that the classic first order irreversible reaction $A \rightarrow B$ takes place in the first case study, while in the second the reaction network is modelled in accordance with the complex van der Vusse reaction mechanism. The performance of the proposed output feedback control laws is evaluated in both cases through numerical simulations and some comparisons with the result in [24] and a classical PI. As main advantage, the control strategy shows the capability to handle input constraints, still maintaining the computational simplicity typical of conventional regulators. The results illustrate that the controllers lead to good performance in terms of overshoot, settling time and control effort.

\section{1 $A \rightarrow B$ Reaction}

Consider the dynamic behavior of a classic example of continuous stirred tank reactor in which a first order, irreversible endothermic or exothermic reaction $A \rightarrow B$ takes place. The reactor is continuously fed by a liquid flow with the temperature $T^{i n}$ containing the reactant $A$ with the concentration $x_{i n}$. In the liquid phase inside the reactor, which is assumed to be of constant volume, the

component $A$ reacts giving the desired product $B$. The control problem is to 

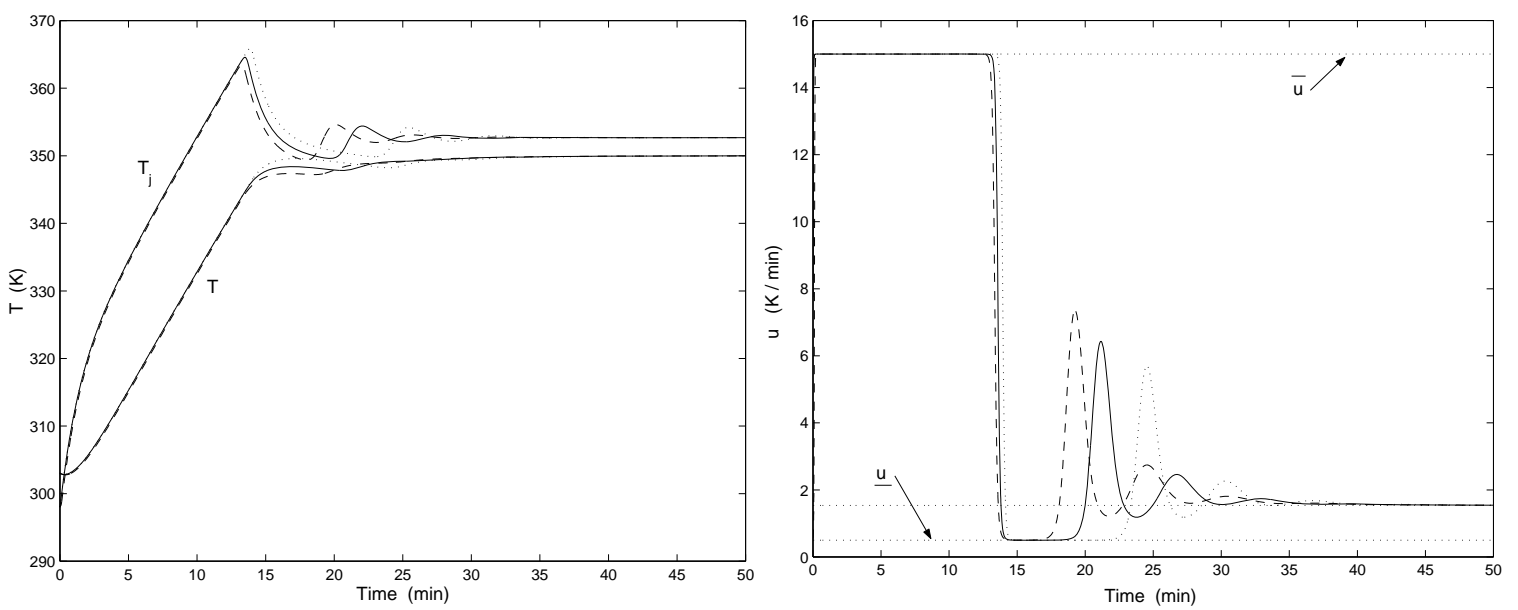

Figure 2: Simulation results for the chemical reactor (69) with endothermic reaction $A \rightarrow B$ when the output feedback control law (70) is active. Reactor and jacket temperature (left) and control action (right) for $k_{\theta}=0.07 \mathrm{~min} /{ }^{\circ} \mathrm{K}$ and different initial values of the controller state $\theta\left({ }^{\circ} \mathrm{K} / \mathrm{min}\right): \theta(0)=1.1 \underline{u}$ (dashed line), $\theta(0)=0.5(\bar{u}+\underline{u})$ (solid line) and $\theta(0)=0.9 \bar{u}$ (dotted line).

perform reactor start-up and operate the reactor at the steady state $\left(T_{s s}, T_{j}^{s s}\right)$ by controlling the inlet fluid flowrate to the jacket. Let $E$ be the activation energy and $k_{0}$ the constant pre-exponential factor. The chemical reaction rate is assumed to follow a first order Arrhenius type kinetic

$$
k(T)=k_{0} \exp \left(-\frac{E}{R T}\right)
$$

and the reactor is modelled in accordance with the Hypothesis $\boldsymbol{H p} \mathbf{1}$ to $\boldsymbol{H p} \boldsymbol{\sigma}$ considered in Section 2.1. In this special case the system (8) becomes

$$
\left\{\begin{aligned}
\dot{x}_{A} & =-k(T) x_{A}+d\left(x_{A}^{i n}-x_{A}\right) \\
\dot{x}_{B} & =k(T) x_{A}+d\left(x_{B}^{i n}-x_{B}\right) \\
\dot{T} & =b k(T) x_{A}+q_{1}\left(T_{j}-T\right)+d\left(T^{i n}-T\right) \\
\dot{T}_{j} & =-q_{2}\left(T_{j}-T\right)+u
\end{aligned}\right.
$$

where $x_{A}$ and $x_{B}$ are the concentrations of the component $A$ and $B$, and simple considerations show that Assumptions 1 to 4 of Section 3 hold. 


\subsubsection{Endothermic Case $(b<0)$}

The output feedback control law (45), namely

$$
\left\{\begin{array}{l}
u=\theta \\
\dot{\theta}=k_{\theta}(\theta-\bar{u})(\theta-\underline{u})\left(T_{j}-T_{j}^{s s}\right)
\end{array}\right.
$$

is implemented on the reactor described by the equations (69) and the closed loop process is simulated, assuming for the model parameters the following values (see [20]): $x_{A}(0)=100 \mathrm{~mol} / \mathrm{m}^{3}, x_{B}(0)=0 \mathrm{~mol} / \mathrm{m}^{3}, T(0)=303^{\circ} \mathrm{K}$, $T_{j}(0)=298^{\circ} \mathrm{K}, k_{0}=1.3677 \times 10^{8} \mathrm{~min}^{-1}, E / R=7290.11^{\circ} \mathrm{K}, d=0.01 \mathrm{~min}^{-1}$, $x_{A}^{i n}=100 \mathrm{~mol} / \mathrm{m}^{3}, x_{B}^{i n}=0 \mathrm{~mol} / \mathrm{m}^{3}, T^{i n}=300^{\circ} \mathrm{K}, b=-0.01196{ }^{\circ} \mathrm{Km}^{3} / \mathrm{mol}$, $q_{1}=0.191 \mathrm{~min}^{-1}, q_{2}=0.574 \mathrm{~min}^{-1}, T_{s s}=350{ }^{\circ} \mathrm{K}, T_{j}^{s s}=352.6^{\circ} \mathrm{K}, x_{A}^{s s}=7.5$ $\mathrm{mol} / \mathrm{m}^{3}, \bar{u}=15^{\circ} \mathrm{K} / \mathrm{min}, \underline{u}=0.5^{\circ} \mathrm{K} / \mathrm{min}$.

Figures 2 and 3 show the jacket and reactor temperatures of the controlled system and the control input behaviour for different initial values of the controller state $\theta$ and for different values of the controller gain $k_{\theta}$, respectively. It should be noted that the manipulated variable $u$ is always bounded between the upper and lower bounds, represented by the dotted lines. Observe also the ability of the controller to stabilise the jacket and reactor temperatures in a relatively short time and the good control performance achieved. It is worthwhile to note that the only information on the model required to implement the controller is the jacket temperature set-point $T_{j}^{s s}$. Finally, the nonlinear controller (70) yields superior regulatory performance (see Figure 4) when compared to a standard saturated PI controller

$$
u(t)=u_{0}+k_{c}\left[e(t)+\frac{1}{\tau_{I}} \int_{0}^{t} e\left(t^{\prime}\right) d t^{\prime}\right]
$$

with $e(t)=T_{j}(t)-T_{j}^{s s}$ and tuning parameters $k_{c}=0.24 \mathrm{~min}^{-1}{ }^{\circ} \mathrm{K}^{-1}$ and $\tau_{I}=3 \mathrm{~min}$.

\subsubsection{Exothermic Case $(b>0)$}

The control of the exothermic reaction $A \rightarrow B$ is achieved by introducing a second control variable. This may be either the reactor feed temperature $T^{\text {in }}$ or the dilution rate $d$. Again it is assumed that the reactor and jacket temperatures are the measurable outputs. If the reactor feed temperature $T^{i n}$ is available for 

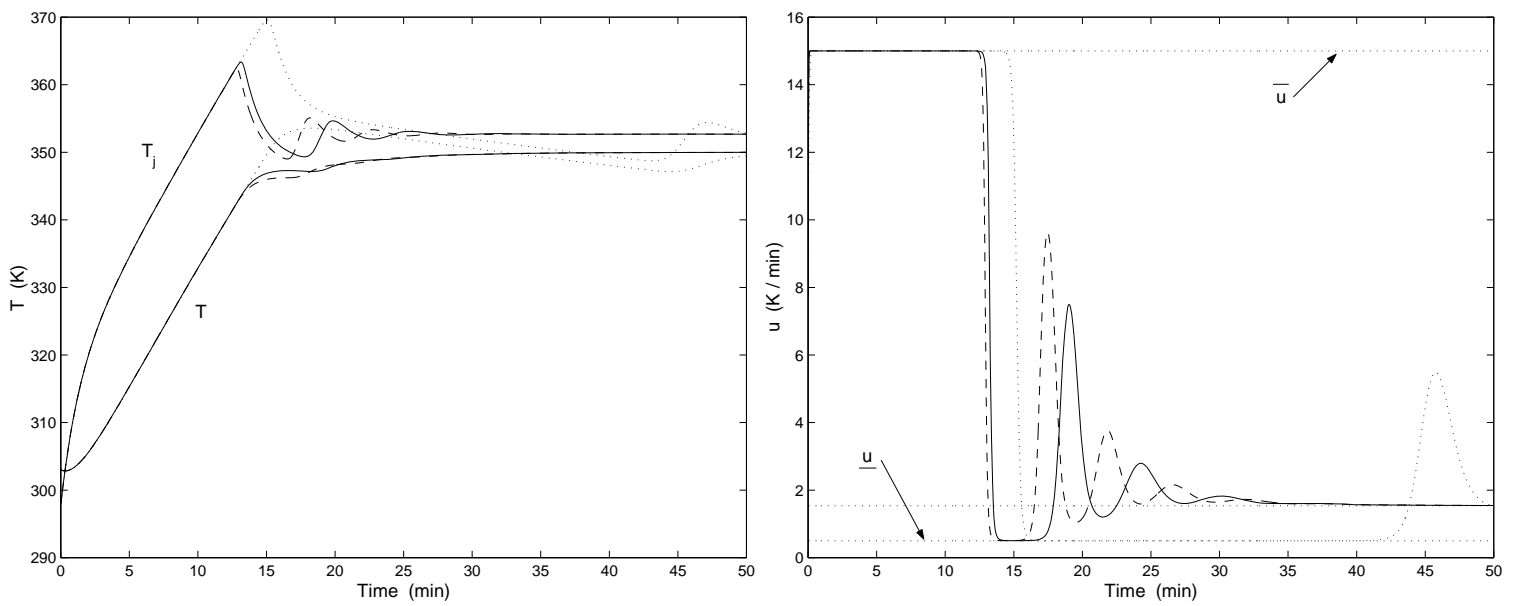

Figure 3: Simulation results for the chemical reactor (69) with endothermic reaction $A \rightarrow B$ when the output feedback control law (70) is active. Reactor and jacket temperature (left) and control action (right) for $\theta(0)=0.5(\bar{u}+\underline{u})$ ${ }^{\circ} \mathrm{K} / \mathrm{min}$ and different values of the controller gain $k_{\theta}\left(\min /{ }^{\circ} \mathrm{K}\right): k_{\theta}=0.03$ (dotted line), $k_{\theta}=0.07$ (solid line) and $k_{\theta}=0.09$ (dashed line).
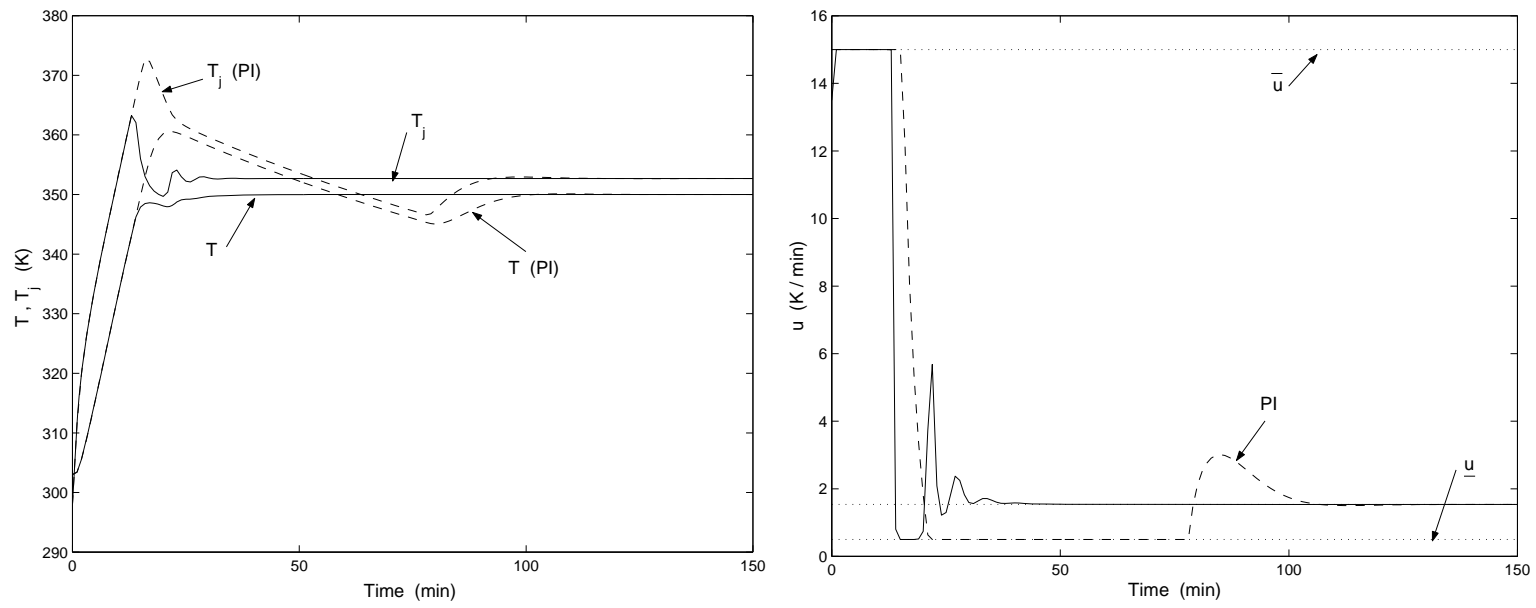

Figure 4: Comparison of performance between the output feedback control law (70) and the PI controller (71) for the chemical reactor (69) with endothermic reaction $A \rightarrow B$ : reactor and jacket temperature (left), control action (right). 
measurements, the controller (58), i.e.

$$
\left\{\begin{aligned}
u & =\theta \\
\dot{\theta} & =k_{\theta}(\theta-\underline{u})(\theta-\bar{u})\left(T_{j}-T_{j}^{s s}\right) \\
T^{i n} & =\gamma-k_{i n}\left(T-T_{s s}\right) \\
\dot{\gamma} & =k_{\gamma}\left(\gamma-\underline{T}^{i n}\right)\left(\gamma-\bar{T}^{i n}\right)\left(T-T_{s s}\right)
\end{aligned}\right.
$$

may be implemented and interpreted as an extension of the dynamic scheme (70) with the addition of a second update law that keeps the estimate of the unknown parameter $T_{s s}^{i n}$ inside the interval limited by the upper and lower bounds $\left[\underline{T}^{i n}, \bar{T}^{i n}\right]$. Alternatively, the dilution rate $d$ may be considered as an additional control variable and the dynamic output feedback controller (68), i.e.

$$
\left\{\begin{aligned}
u & =\theta \\
\dot{\theta} & =k_{\theta}(\theta-\underline{u})(\theta-\bar{u})\left(T_{j}-T_{j}^{s s}\right) \\
d & =v+k_{d}\left[\operatorname{sat}\left(T-T_{s s}\right) \operatorname{sat}\left(T-T^{i n}\right)\right] \\
\dot{v} & =k_{v}\left(T^{i n}-T_{s s}\right)(v-\underline{d})(v-\bar{d})\left(T-T_{s s}\right)
\end{aligned}\right.
$$

implemented. The closed loop system with the controllers (70), (72) and (73) is simulated in order to examine the performance of the controllers in the presence of the input constraints and to show the considerable improvement in the closed loop response for the case of an exothermic reaction when the multivariable controllers (72) and (73) are used. Figures 5, 6 and 7 show the start-up profiles of the measured outputs and manipulated input for the exothermic system (69) when the control input $u$, the control inputs $\left(u, T^{i n}\right)$ and, finally, the control inputs $(u, d)$ are considered. In the presence of tight input constraints, the single input controller (70) shows very poor performance while the controllers (72) and (73) exhibit significantly better performance and faster settling times. The solid, dashed and dotted lines refer to the results of the implementation of the controllers (70), (72) and (73), respectively. For the exothermic reactor, the process parameters are (see $[20]): x_{A}(0)=100 \mathrm{~mol} / \mathrm{m}^{3}, x_{B}(0)=0 \mathrm{~mol} / \mathrm{m}^{3}$, $T(0)=380^{\circ} \mathrm{K}, T_{j}(0)=370^{\circ} \mathrm{K}, \theta(0)=-5^{\circ} \mathrm{K} / \mathrm{min}, \gamma(0)=320^{\circ} \mathrm{K}, v(0)=1.8$ $\mathrm{min}^{-1}, k_{0}=15.155 \times 10^{8} \mathrm{~min}^{-1}, k_{\theta}=0.01 \mathrm{~min} /{ }^{\circ} \mathrm{K}^{2}, k_{\gamma}=0.01{ }^{\circ} \mathrm{K}^{-2} \mathrm{~min}^{-1}$, $k_{v}=0.01{ }^{\circ} K^{-2}, \lambda_{0}=7216.74{ }^{\circ} K, x_{A}^{i n}=100 \mathrm{~mol} / \mathrm{m}^{3}, x_{B}^{i n}=0 \mathrm{~mol} / \mathrm{m}^{3}$, $b=0.00717^{\circ} \mathrm{K} \mathrm{m}^{3} / \mathrm{mol}, q_{1}=0.191 \mathrm{~min}^{-1}, q_{2}=0.574 \mathrm{~min}^{-1}, T_{s s}=350^{\circ} \mathrm{K}$, 


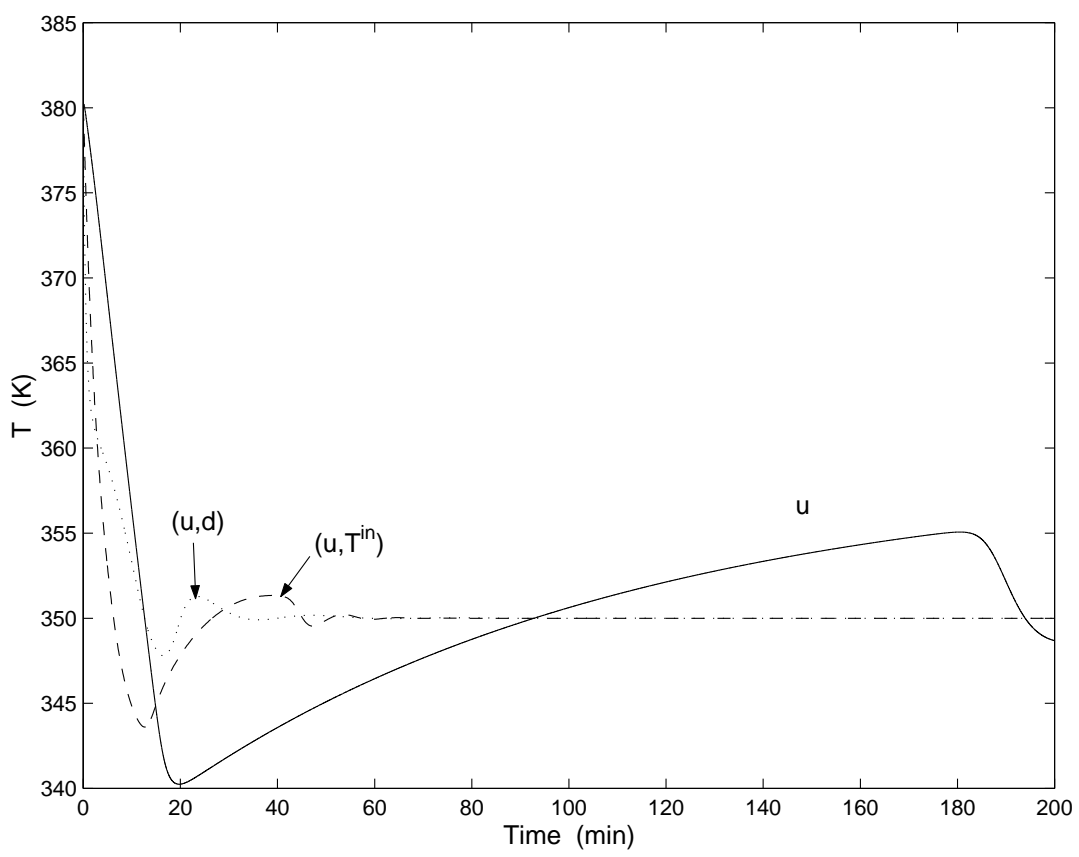

Figure 5: Comparison of the reactor temperature $T$ profiles for the exothermic reaction $A \rightarrow B$ described in (69) with the control laws (70) (solid line), (72) (dashed line) and (73) (dotted line), respectively.

$T_{j}^{s s}=349.19^{\circ} \mathrm{K}, T_{s s}^{i n}=360^{\circ} \mathrm{K}, d_{s s}=0.0144 \mathrm{~min}^{-1}, u_{s s}=-0.4636^{\circ} \mathrm{K} / \mathrm{min}$, $x_{A}^{s s}=0.85 \mathrm{~mol} / \mathrm{m}^{3}, k_{i n}=20, k_{d}=0.0134, \bar{T}_{\text {in }}=400{ }^{\circ} \mathrm{K}, \underline{T}_{i n}=280^{\circ} \mathrm{K}$, $\bar{u}=-0.1^{\circ} \mathrm{K} / \mathrm{min}, \underline{u}=-10^{\circ} \mathrm{K} / \mathrm{min}, \bar{d}=3 \mathrm{~min}^{-1}, \underline{d}=0.001 \mathrm{~min}^{-1}$.

\subsubsection{Comparison between different controllers}

In this section, the controller (70), tested through computer simulations in the previous paragraph, is compared with a similar controller proposed by F. Jadot, G. Bastin and F.Viel [24]. In their work, robust global stabilisation of stirred tank reactors is achieved by means of saturated output feedback control laws. The process considered is again the first order, irreversible exothermic reaction $X_{1} \rightarrow X_{2}$, but the model studied is slightly different from the system (69), since it does not take into account the jacket dynamics. The exothermic reactor is described in this case by a dynamic model of the form

$$
\left\{\begin{aligned}
\dot{x}_{1} & =-r\left(x_{1}, y\right)+d\left(x_{1}^{i n}-x_{1}\right) \\
\dot{x}_{2} & =r\left(x_{1}, y\right)+d\left(x_{2}^{i n}-x_{2}\right) \\
\dot{y} & =\operatorname{\nu r}\left(x_{1}, y\right)-(d+e) y+u
\end{aligned}\right.
$$




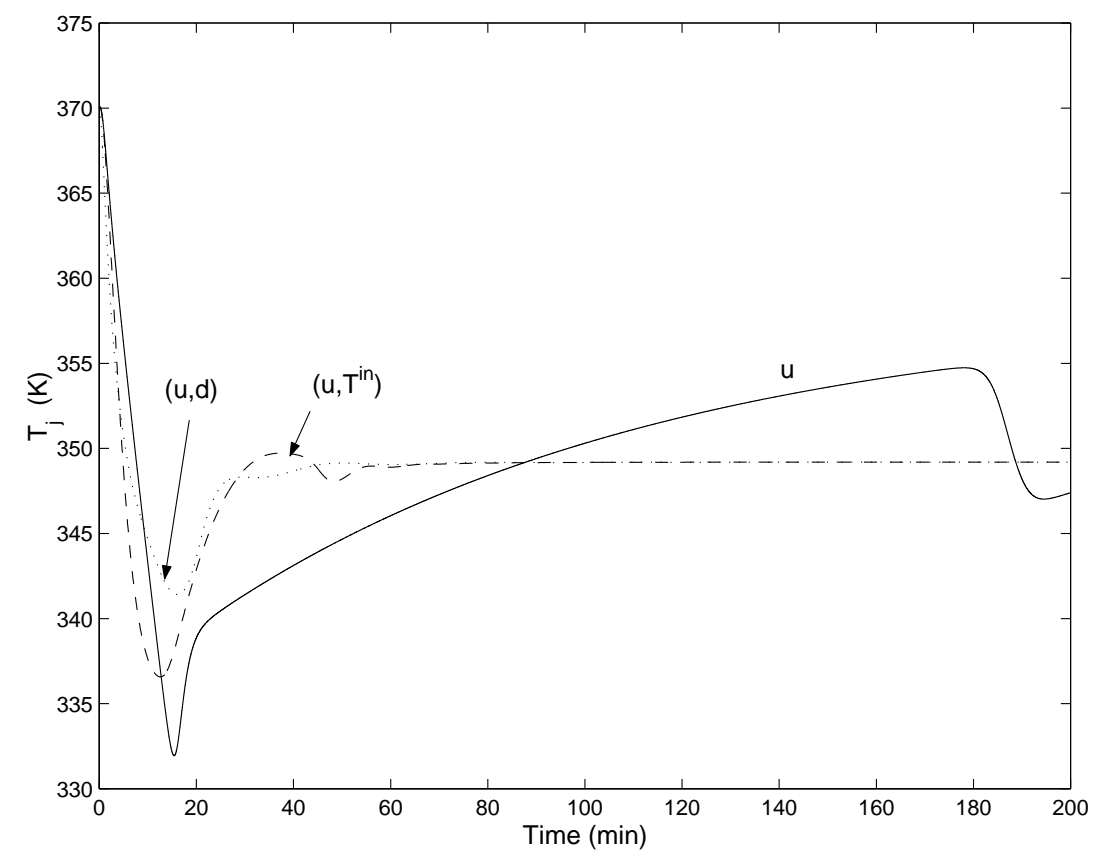

Figure 6: Comparison of the jacket temperature $T_{j}$ profiles for the exothermic reaction $A \rightarrow B$ described in (69) with the control laws (70) (solid line), (72) (dashed line) and (73) (dotted line), respectively.

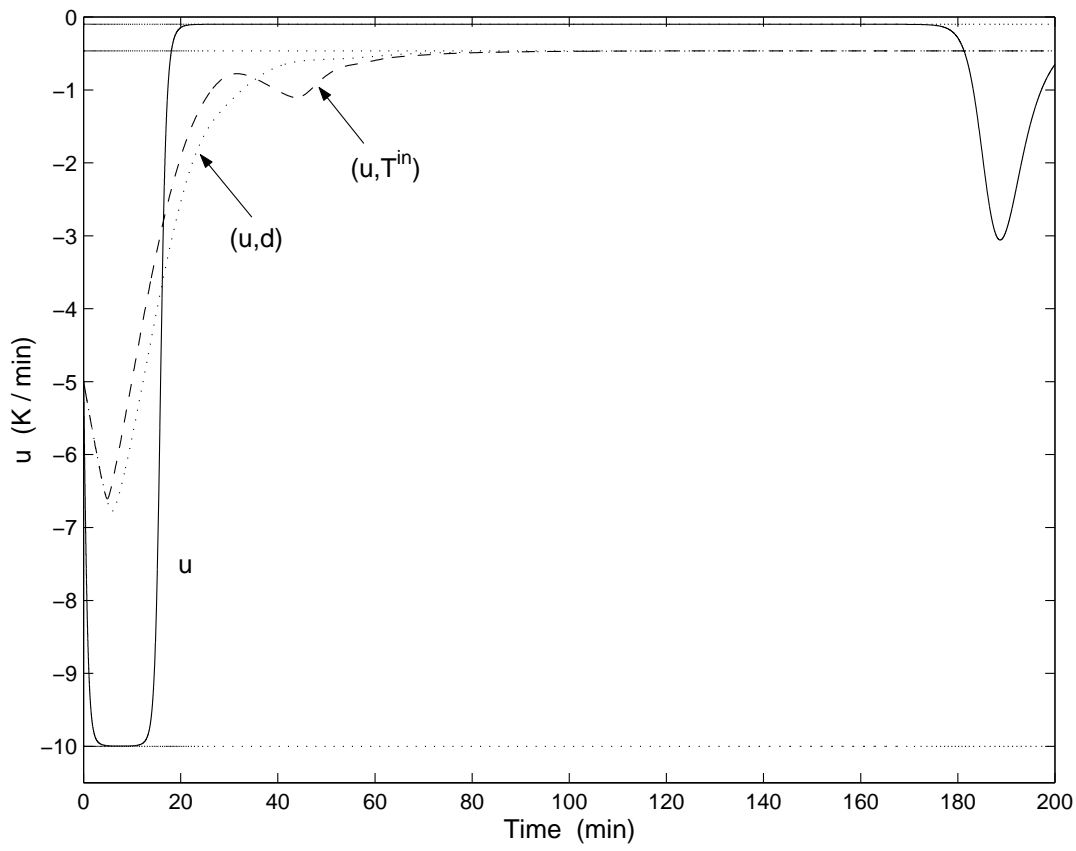

Figure 7: Comparison of the control in put $u$ profiles for the exothermic reaction $A \rightarrow B$ described in (69) with the control laws (70) (solid line), (72) (dashed line) and (73) (dotted line), respectively. 

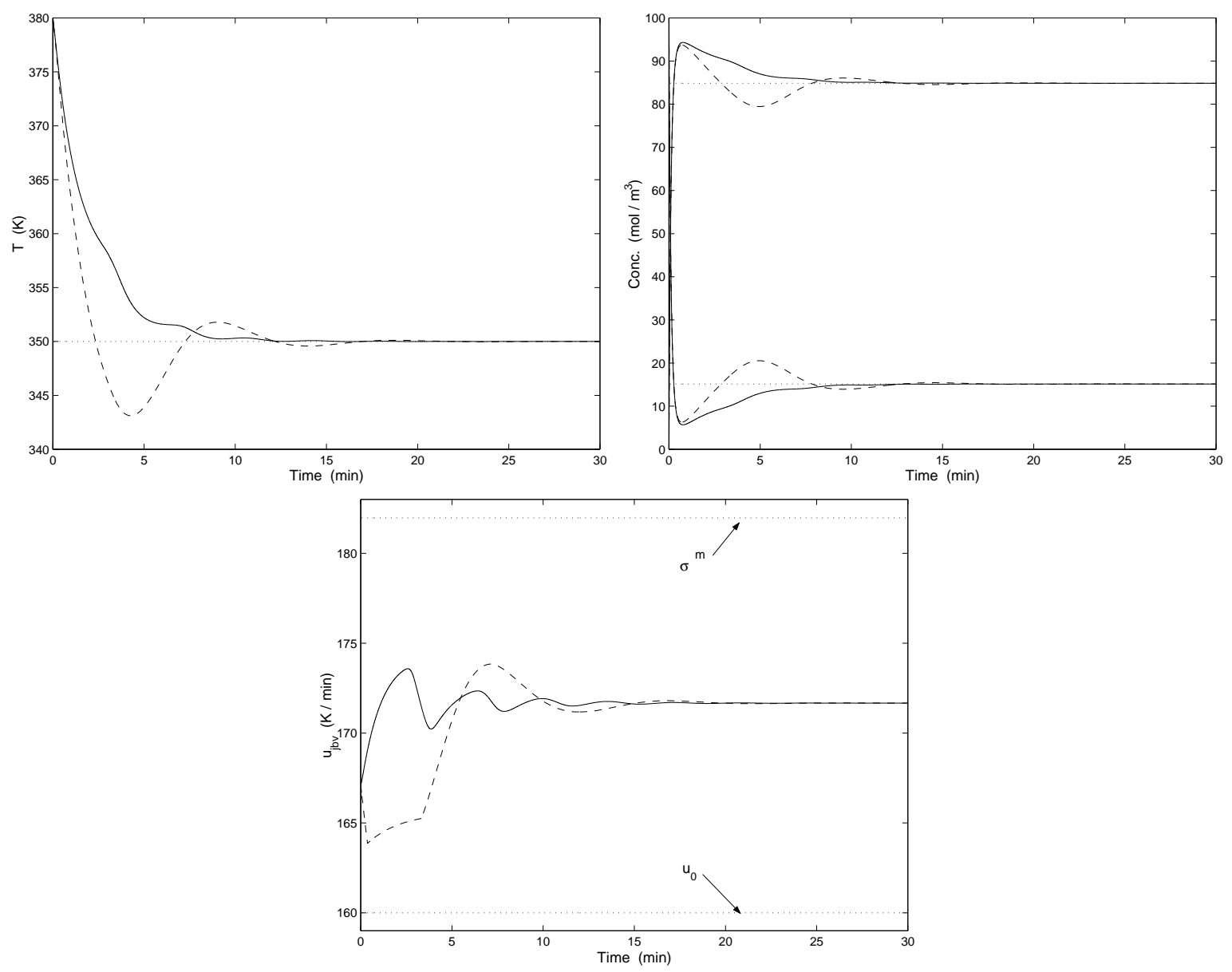

Figure 8: Comparison of performance between the output feedback control law (70) (solid line) applied to control the exothermic first order reaction $A \rightarrow B$ described in (69) and the saturated output feed back controller (76) (dashed line) applied to control the exothermic first order reaction $X_{1} \rightarrow X_{2}$ described in (74): reactor temperature (top left), concentrations (top right) and the control variable $u_{j v b}$ (bottom). 
where $x_{1}$ and $x_{2}$ are the concentrations of the component $X_{1}$ and $X_{2}, r\left(x_{1}, y\right)$ is the reaction rate, expressed by the Arrhenius law

$$
r\left(x_{1}, y\right)=x_{1} \gamma_{1} \exp \left(-\gamma_{2} / y\right)
$$

$y$ is the reactor temperature, $d$ is the dilution rate, $x_{1}^{i n}$ is the reactor feed concentration, $\nu$ is the reaction enthalpy, $e$ is the heat transfer coefficient and $u$ is the manipulated heat.

It is easy to understand the relationship between the symbolism used respectively in system (69) and in system (74). In particular, the manipulated variable selected by Jadot $e t$ al., and renamed here for simplicity $u=u_{j b v}$, is equivalent to

$$
u_{j b v}=q_{1} T_{j}+d T^{i n} .
$$

The saturated output feedback controller proposed by Jadot $e t$ al. is given by

$$
\left\{\begin{array}{l}
u_{j b v}=\operatorname{sat}_{\left[u_{0}, \sigma^{m}\right]}\left(k_{0}+k_{p}\left(y^{*}-y\right)\right)+\operatorname{sat}_{[0,1]}\left(\frac{y^{l}-y}{y^{l}-y^{*}}\right) \operatorname{sat}_{\left[0, \theta^{m}\right]}\left(\theta_{j b v}\right) \\
\dot{\theta}_{j b v}=k_{i}\left(y^{*}-y\right)+\operatorname{sat}_{\left[0, \theta^{m}\right]}\left(\theta_{j b v}\right)-\theta_{j b v}
\end{array}\right.
$$

where $u_{0}, \sigma^{m}, k_{0}, k_{p}, y^{*}, y^{l}, \theta^{m}, k_{i}$ are defined as in [24]. Figure 8 shows the simulation results when the controller $(70)$ and the controller (76) are active. The process parameters considered in the simulation together with the tuning parameters of the controller $(70)$ are: $x_{A}(0)=x_{1}(0)=100 \mathrm{~mol} / \mathrm{m}^{3}, x_{B}(0)=$ $x_{2}(0)=0 \mathrm{~mol} / \mathrm{m}^{3}, T(0)=380^{\circ} \mathrm{K}, T_{j}(0)=276{ }^{\circ} \mathrm{K}, \theta(0)=-40{ }^{\circ} \mathrm{K} / \mathrm{min}$, $\gamma_{1}=15.155 \times 10^{8} \mathrm{~min}^{-1}, k_{\theta}=0.03 \mathrm{~min} /{ }^{\circ} \mathrm{K}^{2}, \gamma_{2}=7216.74^{\circ} \mathrm{K}, x_{A}^{i n}=x_{1}^{i n}=$ $100 \mathrm{~mol} / \mathrm{m}^{3}, x_{B}^{i n}=x_{2}^{i n}=0 \mathrm{~mol} / \mathrm{m}^{3}, b=\nu=0.00717^{\circ} \mathrm{K} \mathrm{m} / \mathrm{mol}, q_{1}=$ $e=0.191 \mathrm{~min}^{-1}, q_{2}=0.574 \mathrm{~min}^{-1}, T_{s s}=y^{*}=350^{\circ} \mathrm{K}, T_{j}^{s s}=300^{\circ} \mathrm{K}$, $T^{i n}=381.2^{\circ} \mathrm{K}, d=0.3 \mathrm{~min}^{-1}, u_{s s}=-28.7^{\circ} \mathrm{K} / \mathrm{min}, x_{A}^{s s}=x_{1}^{s . s}=15.14$ $\mathrm{mol} / \mathrm{m}^{3}, \bar{u}=-25^{\circ} \mathrm{K} / \mathrm{min}$ and $\underline{u}=-57^{\circ} \mathrm{K} / \mathrm{min}$. The design parameters selected for the controller (76) are the following: $\theta_{j b v}(0)=5^{\circ} \mathrm{K} / \mathrm{min}, u_{j b v}^{s s}=$ $q_{1} T_{j}^{s s}+d T^{i n}=171.6^{\circ} \mathrm{K} / \mathrm{min}, u_{0}=150^{\circ} \mathrm{K} / \mathrm{min}, \sigma^{m}=182^{\circ} \mathrm{K} / \mathrm{min}, k_{0}=165$ ${ }^{\circ} \mathrm{K} / \mathrm{min}, k_{p}=0.048 \mathrm{~min}^{-1}, y^{l}=453^{\circ} \mathrm{K}, \theta^{m}=10^{\circ} \mathrm{K} / \mathrm{min}$ and $k_{i}=0.5$ min $^{-2}$.

Observe that the closed loop performance of the two control laws is similar in term of settling time and overshoot. The control objective is effectively achieved in both cases: the reactor temperature converges to the desired set-point and the control action is always bounded. 


\begin{tabular}{|c|c|c|c|}
\hline Variable & Steady State Value & Variable & Steady State Value \\
\hline$x_{A}^{\text {in }}$ & $5.10 \mathrm{~mol} / \mathrm{l}$ & $T$ & $114.2{ }^{\circ} \mathrm{C}$ \\
$x_{A}$ & $2.14 \mathrm{~mol} / \mathrm{l}$ & $T_{j}$ & $112.9{ }^{\circ} \mathrm{C}$ \\
$x_{B}$ & $1.09 \mathrm{~mol} / \mathrm{l}$ & $\dot{V} / V_{R}$ & $14.19 \mathrm{~h}^{-1}$ \\
$T^{\text {in }}$ & $104.9{ }^{\circ} \mathrm{C}$ & $u$ & $-1113.5 \mathrm{~kJ} / \mathrm{h}$ \\
\hline
\end{tabular}

Table 2: Steady state values for the van der Vusse reactor (77).

\subsection{The van der Vusse Reaction}

A more complex example of CSTR dynamics is represented by the van der Vusse reaction, as described in [12]. The main reaction is the transformation of cyclopentadiene (component $A$ ) to the product cyclopentanol (component $B)$. A parallel reaction takes place producing the by-product dicyclopentadiene (component $D$ ). Furthermore, cyclopentenol reacts again giving the undesired product cyclopentandiol (component $C$ ). All these reactions may be described by the reaction scheme

$$
\begin{array}{cccc}
A & \stackrel{k_{1}}{\longrightarrow} & B & \stackrel{k_{2}}{\longrightarrow}
\end{array}
$$

The dynamics of the van der Vusse reactor are simply described by the nonlinear differential equations [12]

$$
\left\{\begin{aligned}
\dot{x}_{A}= & \frac{\dot{V}}{V_{R}}\left(x_{A}^{i n}-x_{A}\right)-k_{1}(T) x_{A}-k_{i n}(T) x_{A}^{2} \\
\dot{x}_{B}= & -\frac{\dot{V}}{V_{R}} x_{B}+k_{1}(T) x_{A}-k_{2}(T) x_{B} \\
\dot{T}= & \frac{\dot{V}}{V_{R}}\left(T^{i n}-T\right)-\frac{1}{\rho C_{p}}\left[k_{1}(T) x_{A} \Delta H_{A B}\right. \\
& \left.+k_{2}(T) x_{B} \Delta H_{B C}+k_{i n}(T) x_{A}^{2} \Delta H_{A D}\right]+\frac{k_{w} A_{R}}{\rho C_{p} V_{R}}\left(T_{j}-T\right) \\
\dot{T}_{j}= & \frac{1}{m_{K} C_{P K}}\left[u+k_{w} A_{R}\left(T-T_{j}\right)\right]
\end{aligned}\right.
$$

As usual, the control input $u$ is the heat removal while the additional control variables are the reactor feed temperature $T^{i n}$ and the dilution rate $d=\frac{\dot{V}}{V_{R}}$ expressed as the flowrate normalised by the reactor volume. These manipulated 


\begin{tabular}{|c|c|c|}
\hline Parameter & Value & Description \\
\hline$k_{10}$ & $1.28710^{12} h^{-1}$ & collision factor for reaction $k_{1}$ \\
\hline$k_{20}$ & $1.28710^{12} h^{-1}$ & collision factor for reaction $k_{2}$ \\
\hline$k_{30}$ & $9.04310^{9} 1 / \mathrm{mol} A h$ & collision factor for reaction $k_{3}$ \\
\hline$E_{1}$ & $-9.758 .3^{\circ} \mathrm{K}$ & activation energy for reaction $k_{1}$ \\
\hline$E_{2}$ & $-9.758 .3^{\circ} \mathrm{K}$ & activation energy for reaction $k_{2}$ \\
\hline$E_{3}$ & $-8560^{\circ} \mathrm{K}$ & activation energy for reaction $k_{3}$ \\
\hline$\Delta H_{A B}$ & $4.2 \mathrm{~kJ} / \mathrm{mol} A \mathrm{~h}$ & enthalpies of reaction $k_{1}$ \\
\hline$\Delta H_{B C}$ & $-11.0 \mathrm{~kJ} / \mathrm{molB} \mathrm{h}$ & enthalpies of reaction $k_{2}$ \\
\hline$\Delta H_{A D}$ & $-41.85 \mathrm{~kJ} / \mathrm{mol} A \mathrm{~h}$ & enthalpies of reaction $k_{3}$ \\
\hline$\rho$ & $0.9342 \mathrm{~kg} / \mathrm{l}$ & density \\
\hline$C_{p}$ & $3.01 \mathrm{~kJ} / \mathrm{kg}^{\circ} \mathrm{K}$ & heat capacity \\
\hline$k_{w}$ & $4032 k J / h m^{2}{ }^{\circ} K$ & heat transfer parameter for cooling jacket \\
\hline$A_{R}$ & $0.215 \mathrm{~m}^{2}$ & surface of cooling jacket \\
\hline$V_{R}$ & $0.01 \mathrm{~m}^{3}$ & reactor volume \\
\hline$m_{K}$ & $5.0 \mathrm{~kg}$ & coolant mass \\
\hline$C_{P K}$ & $2.0 \mathrm{~kJ} / \mathrm{kg}{ }^{\circ} \mathrm{K}$ & heat capacity of coolant \\
\hline
\end{tabular}

Table 3: Physical parameters for the van der Vusse reactor (77).

variables are constrained by

$$
\begin{aligned}
-150 \mathrm{~kJ} / \mathrm{min} & \leq u \leq 0 \mathrm{~kJ} / \mathrm{min} \\
95{ }^{\circ} \mathrm{C} & \leq T^{i n} \leq 130{ }^{\circ} \mathrm{C} \\
0.05 \mathrm{~min}^{-1} & \leq d \leq 0.53 \mathrm{~min}^{-1}
\end{aligned}
$$

The reaction rates $k_{i}$ are functions of the reactor temperature $T$ and follow the Arrhenius law

$$
k_{i}(T)=k_{i 0} \exp \left(\frac{E_{i}}{T+273.15}\right) .
$$

The steady state values together with the values of the physical parameters of the system (77) are listed in Table 2 and Table 3 . It is worth pointing out that, whatever the initial conditions of the system are, the concentration trajectories 


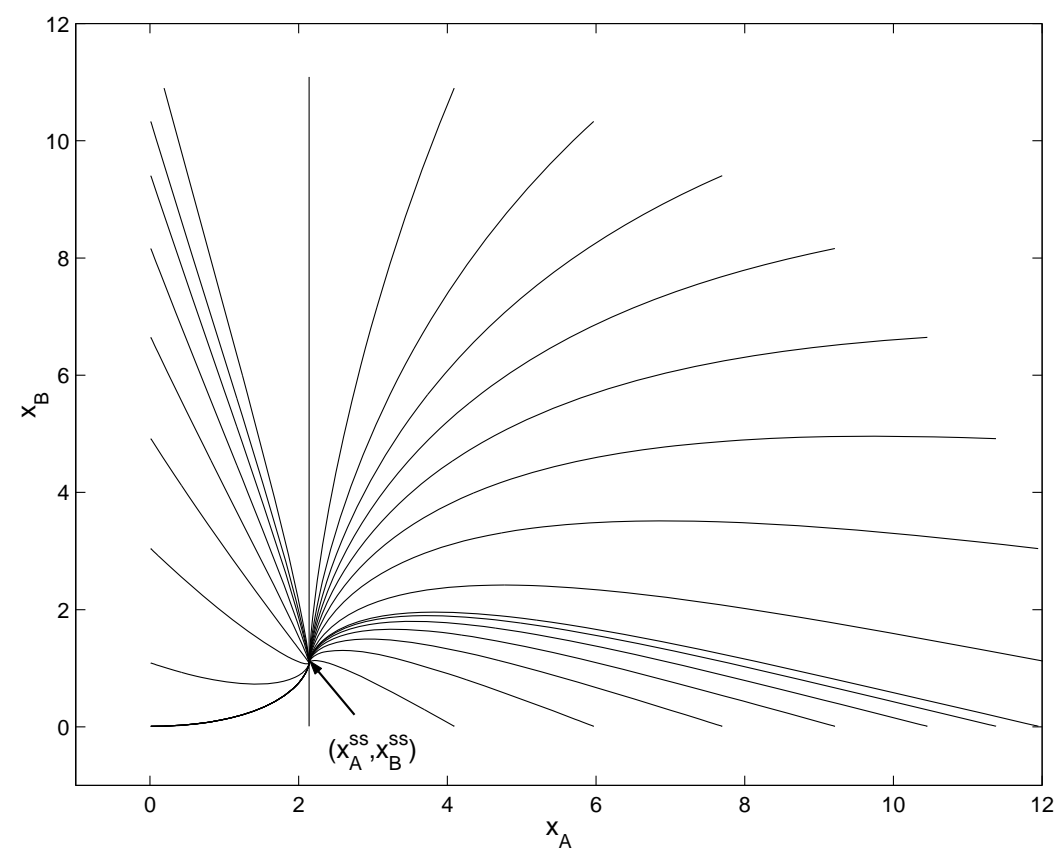

Figure 9: Phase portrait of the isothermal Van der Vusse reactor.

obtained in isothermal condition, i.e. $T=T_{s s}$, for the system

$$
\left\{\begin{aligned}
\dot{x}_{A} & =\frac{\dot{V}}{V_{R}}\left(x_{A}^{i n}-x_{A}\right)-k_{1}\left(T_{s s}\right) x_{A}-k_{i n}\left(T_{s s}\right) x_{A}^{2} \\
\dot{x}_{B} & =-\frac{\dot{V}}{V_{R}} x_{B}+k_{1}\left(T_{s s}\right) x_{A}-k_{2}\left(T_{s s}\right) x_{B}
\end{aligned}\right.
$$

converge all to the same equilibrium point $x_{s s}=\left(x_{A}^{s s}, x_{B}^{s s}\right)$, as it is shown in Figure 9. Hence, the stability properties of this system can be studied by applying the control design proposed in Section 5. The results shown in Figures 10,11, 12 and 13 are obtained when the dynamic output feedback control laws (70), (72) and (73) are implemented. Because of the high rate of heat production by the reaction, the performance of the multivariable controllers (72) and (73) are better than the performance of the single input controller (70). In fact, for the single input controller, the cooling rate goes to its lower bound for a longer period of time during which $T$ and $T_{j}$ cannot be controlled. These simulation results clearly demonstrate the improvement that can be obtained by using additional control variables.

\section{Conclusions}

This work has considered the output feedback regulation of endothermic and exothermic chemical reactors. In particular, the problem of global temperature 


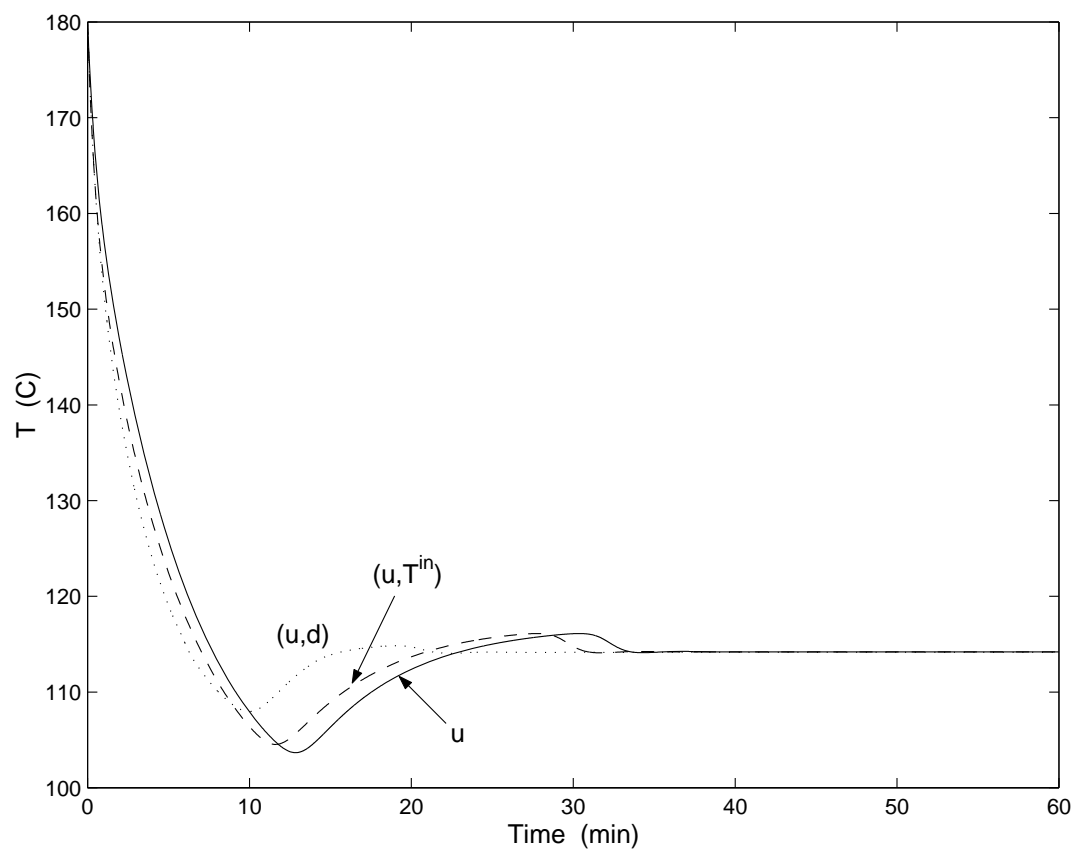

Figure 10: Comparison of the reactor temperature $T$ profiles for the van der Vusse reaction described in (77) with the control laws (70)(solid line), (72) (dashed line) and (73) (dotted line), respectively.

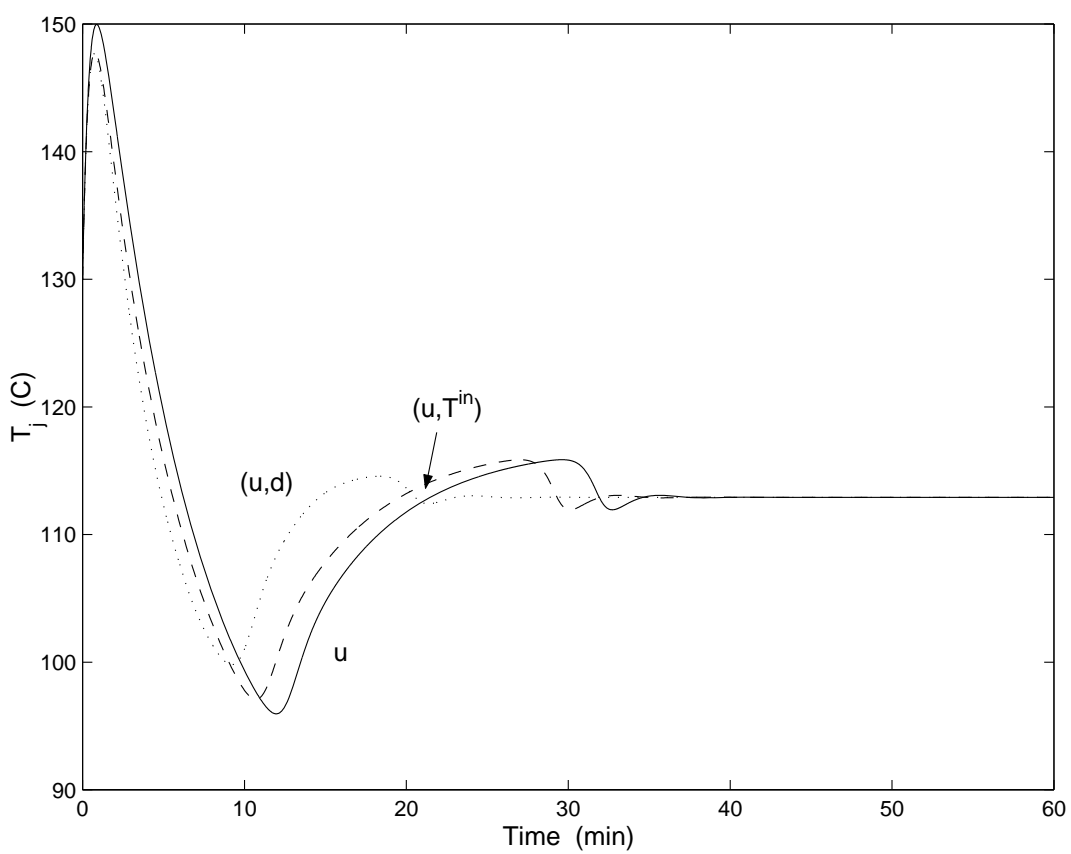

Figure 11: Comparison of the jacket temperature $T_{j}$ profiles for the van der Vusse reaction described in (77) with the control laws (70)(solid line), (72) (dashed line) and (73) (dotted line), respectively. 


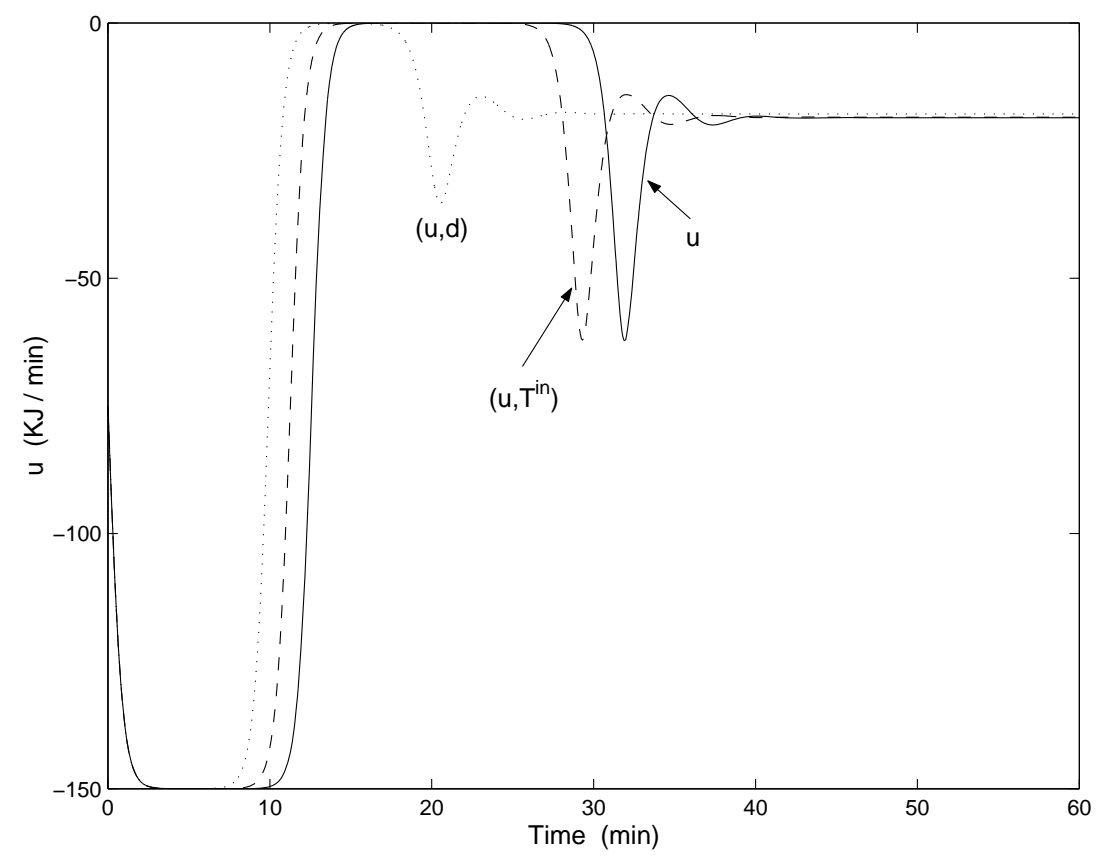

Figure 12: Comparison of the manipulated variable $u$ profiles for the van der Vusse reaction described in (77) with the control laws (70)(solid line), (72) (dashed line) and (73) (dotted line), respectively.

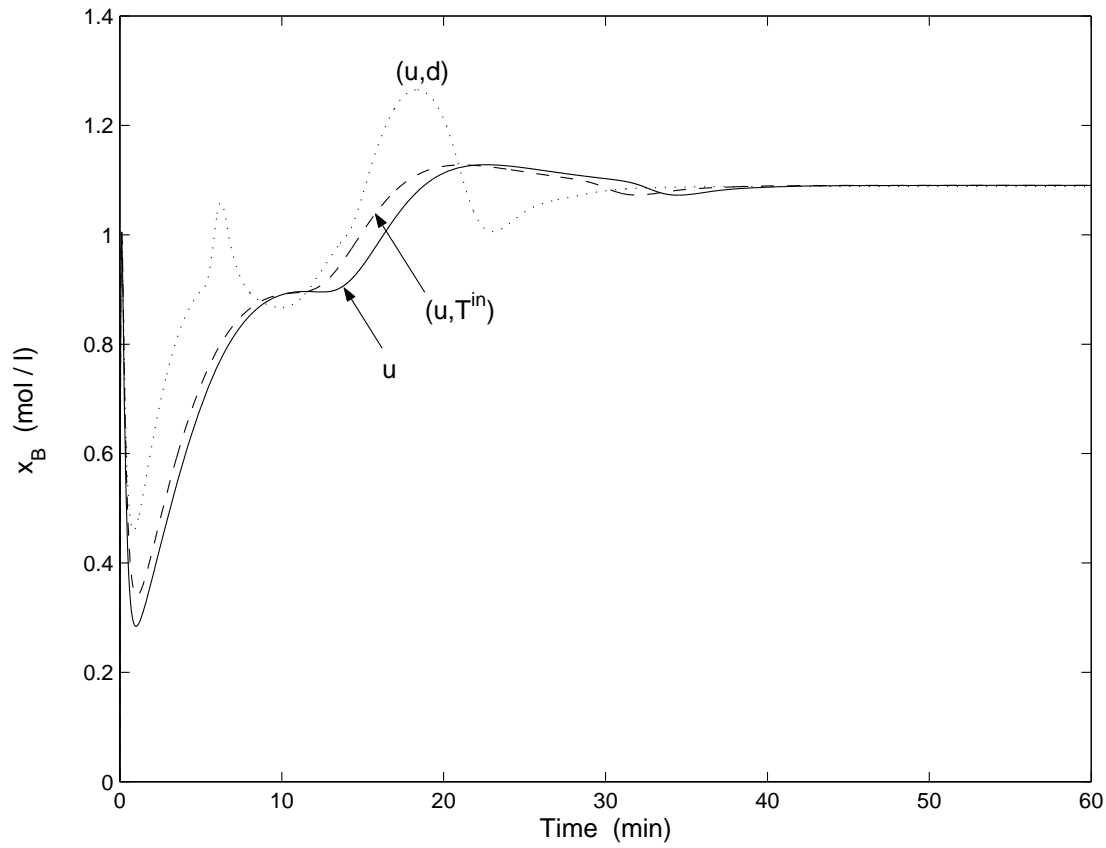

Figure 13: Comparison of the product $\mathrm{B}$ concentration $x_{B}$ profiles for the van der Vusse reaction described in (77) with the control laws (70)(solid line), (72) (dashed line) and (73) (dotted line), respectively. 
control for a general class of chemical reactors has been addressed and solved using Lyapunov techniques. A nonlinear reactor model has been used to synthesize output feedback control laws, i.e. feedback control laws which generate the control signals on the basis of the available measurements only. Under reasonable and easy to test assumptions, global temperature control has been achieved in presence of general and tight control bounds. The proposed approach offers some important advantages including constraint handling capability and computational simplicity: the only condition to be fulfilled is a simple and natural feasibility condition, namely that the steady state control is admissible. The result is that the difficulty in the measurement or the estimation of some state variables has been overcome given that all the proposed controllers are based only on the measured variable. Finally some numerical simulations, illustrating the theoretical results, are presented for a simple and a more complex reaction mechanism together with some comparisons with existing methods. 


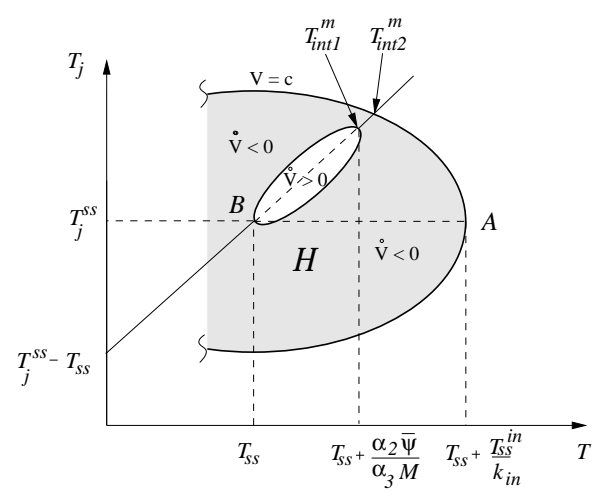

Figure 14: The positively invariant set $\mathcal{H}$.

\section{A Positively Invariant Set $\mathcal{H}$}

Consider the set (see Figure 14)

$$
\mathcal{H}=\left\{\left(T, T_{j}\right) \mid \frac{q_{2}}{2}\left(T-T_{s s}\right)^{2}+\frac{q_{1}}{2}\left(T_{j}-T_{j}^{s s}\right)^{2} \leq \frac{q_{2}}{2}\left(\frac{T_{s s}^{i n}}{k_{i n}}\right)^{2}\right\}
$$

and the function

$$
V=\frac{q_{2}}{2}\left(T-T_{s s}\right)^{2}+\frac{q_{1}}{2}\left(T_{j}-T_{j}^{s s}\right)^{2} .
$$

Moreover, consider the ellipse

$$
V=\frac{q_{2}}{2}\left(T-T_{s s}\right)^{2}+\frac{q_{1}}{2}\left(T_{j}-T_{j}^{s s}\right)^{2}=\frac{q_{2}}{2}\left(\frac{T_{s s}^{i n}}{k_{i n}}\right)^{2}
$$

passing through the point

$$
A=\left(T_{s s}+\frac{T_{s s}^{i n}}{k_{i n}}, T_{j}^{s s}\right)
$$

the ellipse

$$
\begin{aligned}
& \left(q_{1}+d+d k_{i n}-\bar{B}_{2}\right)\left(T-T_{s s}\right)^{2}+q_{1}\left(T_{j}-T_{j}^{s s}\right)^{2}-2 q_{1}\left(T-T_{s s}\right)\left(T_{j}-T_{j}^{s s}\right) \\
& -\left(d+d k_{i n}-\bar{B}_{2}\right) \frac{\alpha_{2}}{\alpha_{3} M} \bar{\psi}\left(T-T_{s s}\right)=0
\end{aligned}
$$

obtained by differentiating with respect to time the equation (79) along the trajectory of the system (49), and the set of straight lines

$$
T_{j}-T_{j}^{s s}=m\left(T-T_{s s}\right)
$$

centered at the point $B=\left(T_{s s}, T_{j}^{s s}\right)$. Let $T_{i n t 1}^{m}$ and $T_{i n t 2}^{m}$ be the "right" intersections of the set of lines (82) with the two ellipses (81) and (80), namely

$$
T_{i n t 1}^{m}=T_{s s}+\frac{\left(d+d k_{i n}-\bar{B}_{2}\right)}{\left(q_{1}+d+d k_{i n}-\bar{B}_{2}+q_{1} m^{2}-2 q_{1} m\right)} \frac{\alpha_{2}}{\alpha_{3} M} \bar{\psi}
$$


and

$$
T_{i n t 2}^{m}=T_{s s}+\frac{T_{s s}^{i n}}{k_{i n}} \sqrt{\frac{q_{2}}{q_{2}+q_{1} m^{2}}} .
$$

To avoid the intersection of the two ellipses (81) and (80) it is necessary that

$$
T_{i n t 1}^{m} \leq T_{i n t 2}^{m}
$$

for all $m$, namely

$$
\frac{\left(d+d k_{i n}-\bar{B}_{2}\right)}{\left(q_{1}+d+d k_{i n}-\bar{B}_{2}+q_{1} m^{2}-2 q_{1} m\right)} \frac{\alpha_{2}}{\alpha_{3} M} \bar{\psi} \leq \frac{T_{s s}^{i n}}{k_{i n}} \sqrt{\frac{q_{2}}{q_{2}+q_{1} m^{2}}} .
$$

This is equivalent to a condition over the tuning parameter $k_{i n}$, i.e.

$$
k_{i n} \leq \frac{\alpha_{3} M T_{s s}^{i n}}{\alpha_{2} \bar{\psi}} \frac{\left(q_{1}+d+d k_{i n}-\bar{B}_{2}+q_{1} m^{2}-2 q_{1} m\right)}{\left(d+d k_{i n}-\bar{B}_{2}\right)} \sqrt{\frac{q_{2}}{q_{2}+q_{1} m^{2}}}
$$

Note now that

$$
\frac{\left(q_{1}+d+d k_{i n}-\bar{B}_{2}+q_{1} m^{2}-2 q_{1} m\right)}{\sqrt{q_{2}+q_{1} m^{2}}} \geq \frac{\left(d+d k_{i n}-\bar{B}_{2}\right)}{\sqrt{q_{2}+q_{1}}} \quad \forall m>0
$$

hence (85) holds if

$$
k_{i n} \leq \frac{T_{s s}^{i n}}{\bar{\psi}} \frac{\alpha_{3}}{\alpha_{2}} M \sqrt{\frac{q_{2}}{q_{1}+q_{2}}}
$$

This concludes the proof. 


\section{References}

[1] A. K. Adebekun and F. J. Schork. On the robust global stabilization of nth order reactions. Chem. Eng. Commun., 101:1-15, 1991.

[2] K. Adebekun. The robust global stabilisation of a stirred tank reactor. A IChE Journal, 38(5):651-659, 1992.

[3] J. Alvarez, J. Alvarez, and E. Gonzales. Global nonlinear control of a continuous stirred reactor. Chemical Engineering Science, 44(5):1147-1160, 1989.

[4] J. Alvarez, J. Alvarez, and R. Suarez. Nonlinear bounded control for a class of continuous agitated tank reactors. Chemical Engineering Science, 46(12):3235-3249, 1991.

[5] J. Alvarez-Ramirez. Stability of a class of uncertain continuous stirred chemical reactors with a nonlinear feedback. Chemical Engineering Science, 49(11):1743-1748, 1994.

[6] J. Alvarez-Ramirez and R. Femat. Robust PI stabilization of a class of chemical reactors. Systems and Control Letters, 38(4):219-225, 1999.

[7] J. Alvarez-Ramirez, R. Suarez, and R. Femat. Control of continuous-stirred tank reactors: stabilization with unknown reaction rates. Chemical Engineering Science, 51(17):4183-4188, 1996.

[8] R. Antonelli and A. Astolfi. Global temperature stabilization of chemical reactors with bounded control. Proc. AIChE Annual Meeting, November 15-20, Miami Beach, Florida, USA, 1998.

[9] R. Antonelli and A. Astolfi. Global temperature stabilization of continuous stirred tank reactors with bounded output feedback control. Proc. 6th Mediterranean Control Conference, June 9-11, Alghero, Italy, 1998.

[10] R. Antonelli and A. Astolfi. Adaptive output feedback stabilization of a class of uncertain continuous stirred tank reactors. Proc. 14th IFAC World Congress, Beijing, China, 1999.

[11] R. Aris and N. Amundson. An analysis of chemical reactor stability and control. Chemical Engineering Science, 7(8):121-131, 1958. 
[12] H. Chen, A. Kremling, and F. Allgower. Nonlinear predictive control of a benchmark CSTR. Proceedings of 3rd European Control Conference, pages 3247-3252, Rome, Italy, 1995.

[13] Y.-S Chou and W. Wu. Robust controller design for uncertain nonlinear systems via feedback linearization. Chem. Eng. Sci., 50:1429-1439, 1995.

[14] J.M. Coulson, J.F. Richardson, J.R. Backhurst, and J.H. Harker. Coulson and Richardson's chemical engineering. Volume 1: fluid flow, heat transfer and mass transfer. Butterworth-Heinemann, Oxford, Boston, 1999.

[15] P. Daoutidis and C. Kravaris. Dynamic output feedback control of minimum-phase nonlinear processes. Chem. Eng. Sci., 47:837-843, 1992.

[16] D. Dochain. Design of adaptive linearizing controllers for non-isothermal reactors. Int. J. Control, 59:689-710, 1994.

[17] F.J. Doyle III, A.K. Packard, and M. Morari. Robust controller design for a nonlinear CSTR. Chemical Engineering Science, 44(9):1929-1947, 1989.

[18] J.H Espenson. Chemical kinetics and reaction mechanisms. 2nd ed., McGraw-Hill, New York; London, 1995.

[19] A. Fradkov, R. Ortega, and G. Bastin. Semi-adaptive control of convexly parameterized systems with application to temperature regulation of chemical reactors. European Control Conference, 1997.

[20] A.M. Gibon-Fargeot, H. Hammouri, and F. Celle. Nonlinear observers for chemical reactors. Chemical Engineering Science, 49(14):2287-2300, 1994.

[21] C.G. Hill. An introduction to chemical engineering kinetics and reactor design. John Wiley and Sons, New York, 1977.

[22] K. A. Hoo and J. C. Kantor. An exothermic continuous stirred tank reactor is feedback equivalent to a linear system. Chem. Eng. Commun., 37:1-10, 1985 .

[23] F. Jadot. Dynamics and robust nonlinear PI control of stirred tank reactors. Ph.D. Thesis, CESAME, Universite Catholique de Louvain, Belgium, 1996.

[24] F. Jadot, G. Bastin, and F. Viel. Robust global stabilisation of stirred tank reactors by saturated output feedback. European Journal of Control, 5:361-371, 1999. 
[25] H. K. Khalil. Nonlinear systems. 2nd Edition, Prentice Hall, Upper Saddle River, New Jersey, 1996.

[26] H.K. Khalil and F. Esfandiari. Semiglobal stabilization of a class of nonlinear systems using output feedback. IEEE Transactions on Automatic Control, 38(9):1412-1415, 1993.

[27] P. Kittisupakorn. The use of nonlinear model predictive control techniques for the control of a reactor with exothermic reactions. Ph.D. Thesis, Chemical Engineering Department, Imperial College of Science, Technology and Medicine, London, U.K., 1996.

[28] K. A. Kosanovich, M. J. Piovoso, V. Rokhlenko, and A. Guez. Nonlinear adaptive control with parameter estimation of a CSTR. J. Proc. Contr., 5:133-148, 1995.

[29] C. Kravaris and C.-B. Chung. Nonlinear state feedback synthesis by global input/output linearization. AIChE Journal, 33:592-599, 1987.

[30] M.J. Kurtz and M.A. Henson. Input-output linearizing control of constrained nonlinear processes. Journal of Process Control, 7(1):3-17, 1997.

[31] L. C. Limqueco and J. C. Kantor. Nonlinear output feedback control of an exothermic reactor. Computers Chem. Eng., 14:427-437, 1990.

[32] M. Morari and E. Zafiriou. Robust Process Control. Prentice-Hall, Englewood Cliffs, NJ, 1989.

[33] R. H. Perry and D. Green. Perry's Chemical Engineers' Handbook. Sixth Edition, McGraw-Hill, Tokio; London, 1984.

[34] A. Uppal, W.H. Ray, and A.B. Poore. On the dynamic behaviour of continuous stirred tank reactors. Chem. Eng. Sci., 29:967-985, 1976.

[35] F. Viel, F. Jadot, and G. Bastin. Global stabilization of exothermic chemical reactor under input constraints. Automatica, 33(8):1437-1448, 1997.

[36] F. Viel, F. Jadot, and G. Bastin. Robust feedback stabilization of chemical reactors. IEEE Trans. Autom. Control, 42(4):473-481, 1997. 